\title{
Characteristics of electric vehicle charging demand at multiple types of location- Application of an agent-based trip chain model
}

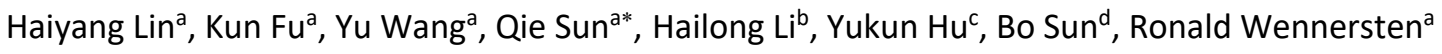 \\ ${ }^{a}$ Institute of Thermal Science and Technology, Shandong University, Jingshi Road No.17923, Jinan and 250061, China \\ ${ }^{b}$ School of Business, Society and Technology, Mälardalen University, Sweden \\ c Department of Civil, Environmental \& Geomatic Engineering, University College London, London \\ ${ }^{d}$ School of Control Science and Engineering, Shandong University, Jinan, 250061, China
}

\section{Abstract}

This paper developed an agent-based trip chain model (ABTCM) to study the distribution of electric vehicles (EVs) charging demand and its dynamic characteristics, including flexibility and uncertainty, at different types of location. Key parameters affecting charging demand include charging strategies, i.e. uncontrolled charging (UC) and off-peak charging (OPC), and EV supply equipment, including three levels of charging equipment.

The results indicate that the distributions of charging demand are similar as the travel patterns, featured by traffic flow at each location. A discrete peak effect was found in revealing the relation between traffic flow and charging demand, and it results in the smallest equivalent daily charging demand and peak load at public locations. EV charging and vehicle-to-grid (V2G) flexibility were examined by instantaneous adjustable power and accumulative adjustable amount of electricity. The EVs at home locations have the largest charging and V2G flexibility under the UC strategy, except for a period of regular working time. The V2G flexibility at work and public locations is generally larger than charging flexibility. Due to the fast charging application, the uncertainties of charging demand at public locations are the highest in all locations. In addition, the OPC strategy mitigates the uncertainty of charging demand.

\section{Keywords:}

Electric vehicle; Agent-based trip chain model; Vehicle to grid; Fast charging; Charging flexibility

\begin{tabular}{|ll}
\hline Abbreviations & \\
& \\
ABTCM & Agent-based trip chain model \\
EV & Electric vehicle \\
UC & Uncontrolled charging \\
OPC & Off-peak charging \\
V2G & Vehicle to grid \\
NHTS & National Household Travel Survey \\
EVA & Electric vehicle agent \\
EVSE & Electric vehicle supply equipment \\
TPM & Transfer probability matrix
\end{tabular}

* Qie Sun. Tel.: +86-531-88399598; E-mail address: qie@sdu.edu.cn 


\begin{tabular}{|ll|} 
PDF & Probability density function \\
RMSE & Root-mean-square error \\
CC & Correlation coefficient \\
TOU & Time-of-use \\
ARMSE & Average root-mean-square error \\
SOC & State-of-charge \\
ARMSE & Average root-mean-square error \\
TCD & Total charging demand \\
HCD & Home location charging demand \\
WCD & Work location charging demand \\
PCD & Public location charging demand \\
RCD & Recreation purpose charging demand \\
SCD & Shopping purpose charging demand \\
MCD & Meal purpose charging demand \\
EDCD & Equivalent daily charging demand \\
EDPL & Equivalent daily peak load \\
GIS & Geographic information system \\
\hline
\end{tabular}

\section{Introduction}

The rising concerns about fossil fuel depletion and climate change have motivated the innovation of clean technologies. Electric vehicle (EV) is regarded as a promising solution to energy security and reduction in greenhouse gas emissions [1]. With the motivation of government policies and automaker investments, the fleet of EVs and the supporting infrastructure has been rapidly growing in recent years [2]. The growing penetration of EV poses both challenges and opportunities to the operation of power grids. On one hand, electric grids capacity may not be sufficient for the EV loads, especially when peak charging demand occurs [3]. On the other, the operation of power grids can be improved by effectively managing the charging and discharging of EVs, while increasing the integration of intermittent renewable energy [4] and providing ancillary power services [5].

In order to analyze the impacts of EVs, it is important to understand the distribution and flexibility of EVs charging demands, especially the respective characteristics at different types of locations, which are crucial information for grid operation [6], renewable energy integration [7], intra-city charging station planning [8] and energy management of EV batteries [9, 10]. To this objective, a reliable model capable of simulating the travel patterns of a large fleet of EVs and their charging demand at different locations is necessary.

In existing studies, the spatial and temporal distributions of EVs charging demand are associated with different facilities. A majority of early studies have used a deterministic approach to model EVs charging demands. In [11], the EVs, which participated in strategic energy management of microgrids as energy storage units, were assumed to take three $40 \mathrm{~km}$ trips in one day. In [12], the EVs always charged at 18:30 and interacted with a micro-cogeneration system. The impact of vehicle to grid (V2G) on reducing peak demand of energy markets was analyzed in [13], where all EVs were available for $\mathrm{V} 2 \mathrm{G}$ at 18:00. In addition, other approaches derived static charging 
patterns using annual travel data [14] or average values [15] rather than expected charging time and demands. These deterministic models may be useful for evaluating long-term impacts of EV [16], while are not so useful for short-term management/control [17].

For a better understanding and recurrence of EVs' travel patterns and charging characteristics, existing studies developed several prototype trip models, which are able to capture the main characteristics of travel patterns, but can hardly reflect the stochastic characteristics of individual trips and associated energy consumption. Fernandes et al. [18] performed a mid-term simulation of a Spanish power system considering EV penetration with five types of travel pattern. Four types of EV fleets with different number of EVs, travel time and desired $\mathrm{SOC}$ were studied as intermediate agents between end-users and distributed renewable generation [19]. Similar models were used to evaluate the impacts of EVs charging on greenhouse gas emissions [20], voltage stabilization in an islanded microgrid [21] and frequency control of grids [22].

In contrast to deterministic models, stochastic modelling has gradually received growing attention. [23] simulated EV charging and V2G using a stochastic travel model and evaluated the impacts on the reliability of a smart grid. [24] quantified the potential of EVs to consume renewable energy and highlighted the importance of trip information as regards managing EV flexibility. However, existing stochastic models are usually based on home-related parameters: time of departure from home, time of arriving at home and daily travel distance, which can be used to determine an overall amount of energy consumption, while ignoring the variations at various locations. In [25], the three mentioned parameters are used to model EVs stochastic travel and they all assumed that the EVs are charged once per day at home. In reality, EVs can be charged at different kinds of location, such as: residential, commercial, recreation areas and workplaces [26]. In addition, the charging at one location will influence the charging at other locations, since the charging and discharging of batteries take time. Therefore, a detailed loop model considering the variations in EVs location and temporal distribution is important for modelling EVs charging and estimating associated flexibility.

Using real-world data, [27-29] modelled travel patterns at multi-locations and number of daily trips and number of back home times were vital variables. However, those studies did not consider the possibilities of charging and trip purpose transition at different locations. As a result, those models often have a large simulation error, e.g. the error of trip type is about 15\% in [28]. Therefore, a detailed trip chain model considering the location variation and temporal distribution is essential for studying the charging demand of EVs and related characteristics.

The aim of this paper is to analyze the distribution of charging demand and the flexibility of charging and V2G of EVs at different types of location. To achieve this, a novel agent-based trip chain model (ABTCM), which considered various trip purposes and locations, was developed to simulate the travel patterns of EVs. The ABTCM was validated using the data from the American National Household Travel Survey (NHTS) [30]. The distributions of charging demand at three types of location were studied under two charging strategies, namely the uncontrolled charging (UC) strategy and the off-peak charging (OPC) strategy. The influence of traffic flow on charging demand was then specifically investigated. To evaluate the flexibility of charging and V2G of EVs, the paper calculated maximum adjustable charging/V2G power, which reflects the instantaneous capacity of load shifting, and maximum adjustable amount of charging/V2G electricity, which reflects the aggregate amount of load that can be shifted. The paper also evaluated the uncertainty of charging demand. 
The contributions of the paper are two-fold. Firstly, the ABTCM model, which captures the characteristics of vehicle usage, is able to simulate the heterogeneous travel patterns and charging patterns of EVs with a higher simulation accuracy than existing studies. The model provides a generic method for studying complex transportation systems and associated energy consumption. Secondly, owing to the novel ABTCM, the dynamic characteristics of energy demand at different kinds of location were comprehensively investigated. The results provide important information on how charging stations should be planned and how demand-side energy management, if possible, could be implemented to satisfy the energy needs at different locations.

\section{Agent-based trip chain model}

This study developed an ABTCM to simulate and analyze the travel and charging patterns of EVs, which are represented by EV agents (EVAs) in the model. The trip chain model specifies three kinds of locations, where EVs park and charge, and seven trip purposes that are performed at the three locations (Fig. 1). The study focused on the most common parking locations and trip purposes in order to obtain generic knowledge for wide energy application. The model differentiated EVs travel and charging on weekdays and on weekends to generate comprehensive results related to travel patterns.

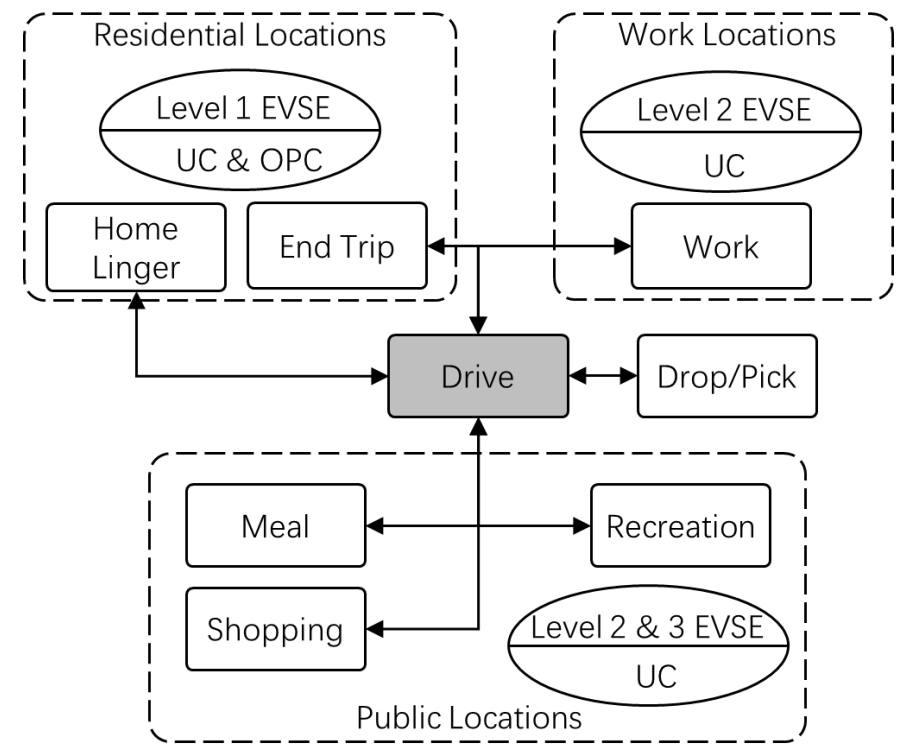

Fig.1 Overview of the EV agent

In the ABTCM, two sub models: the trip chain model and the charging model, work together and define the actions of EVAs. The trip chain model determines the states of EVAs, the rules of states switching and other actions like parking. The charging model controls EVAs' charging and two strategies: uncontrolled charging (UC) and (off-peak charging) OPC, are considered in the model.

\subsection{Trip chain model}

\subsubsection{Real-world data}

The 2017 NHTS [30] contains 514,132 vehicle travel logs by all modes for 24-hour on weekdays and weekends and thus represents the best available source for modelling travel patterns and related impacts. The trip-chain model includes six trip purposes (Table 1), which were retrieved from the dataset and account for near $97 \%$ of private trips and $96 \%$ of all trips. For the convenience 
of modelling, the "home" purpose was further divided into two purposes: "home linger" (P1), which means the EV will leave from home again in the same day and "end trip" (P7).

Table 1. Trip purposes

\begin{tabular}{|c|c|c|c|c|c|c|c|}
\hline Purpose & Home & Work & Shopping & Pick/Drop & Meal & Recreation & Total \\
\hline Symbol & P1/P7 & $\mathrm{P} 2$ & P3 & P4 & P5 & P6 & / \\
\hline Total trip (\%) & 34.5 & 14.4 & 19.9 & 6.3 & 7.8 & 12.9 & 95.8 \\
\hline Private vehicle trip (\%) & 35.4 & 14.1 & 21.2 & 7.3 & 7.8 & 10.9 & 96.7 \\
\hline
\end{tabular}

\subsubsection{Trip chain generation}

The trip chain module generates a series of random trip chains, e.g. starting from home, continuing with one or more trips between different locations and finally ending at home. The electricity demand and time for charging are dependent on the trip, which is characterized by a series of parameters: departure place $\left(p_{d}\right)$, departure time $\left(T_{d}\right)$, trip driving time $\left(t_{d}\right)$, trip driving distance $\left(d_{t}\right)$, destination $\left(p_{e}\right)$ and parking time $\left(t_{p}\right) \cdot T_{d}$ and $p_{d}$ are calculated as Formula 1 and 2 . The entire trip chain is represented by Formula 3. Parameter $n$ in Formula 1-3 represents the $n^{\text {th }}$ trip in the entire trip chain. The trip parameters follow the probabilities calculated from the NHTS dataset.

$$
\begin{array}{cc}
T_{d, n+1}=T_{d, n}+t_{d}+t_{p} \quad(\mathrm{n}=1,2,3 \ldots) \\
p_{d, n+1}=p_{e, n} & (\mathrm{n}=1,2,3 \ldots) \\
\left(p_{d, 1}, p_{e}, T_{d, 1}, t_{d}, t_{p}, d_{t}\right)= & \\
\left(p_{d, 1} ; p_{e, 1}, p_{e, 2} \cdots p_{e, n} ; T_{d, 1} ; t_{d, 1}, t_{d, 2} \cdots t_{d, n} ; t_{p, 1}, t_{p, 2} \cdots t_{p, n} ; d_{t, 1}, d_{t, 2} \cdots d_{t, n}\right)
\end{array}
$$

Every trip chain starts from the first departure from home at time $T_{d, 1}$. The probability density function (PDF) and the related distribution of the time for the first departure are shown in Fig. 2. The parameters of a trip and its conditional probability in a trip chain are dependent on the trip prior to it.

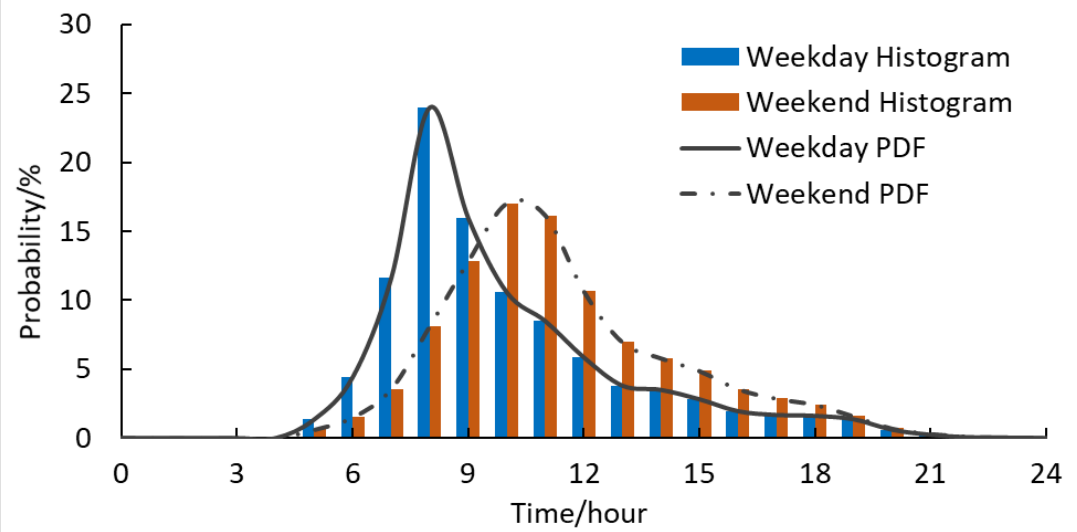

Fig.2 Distribution of the departure time of the first trip 


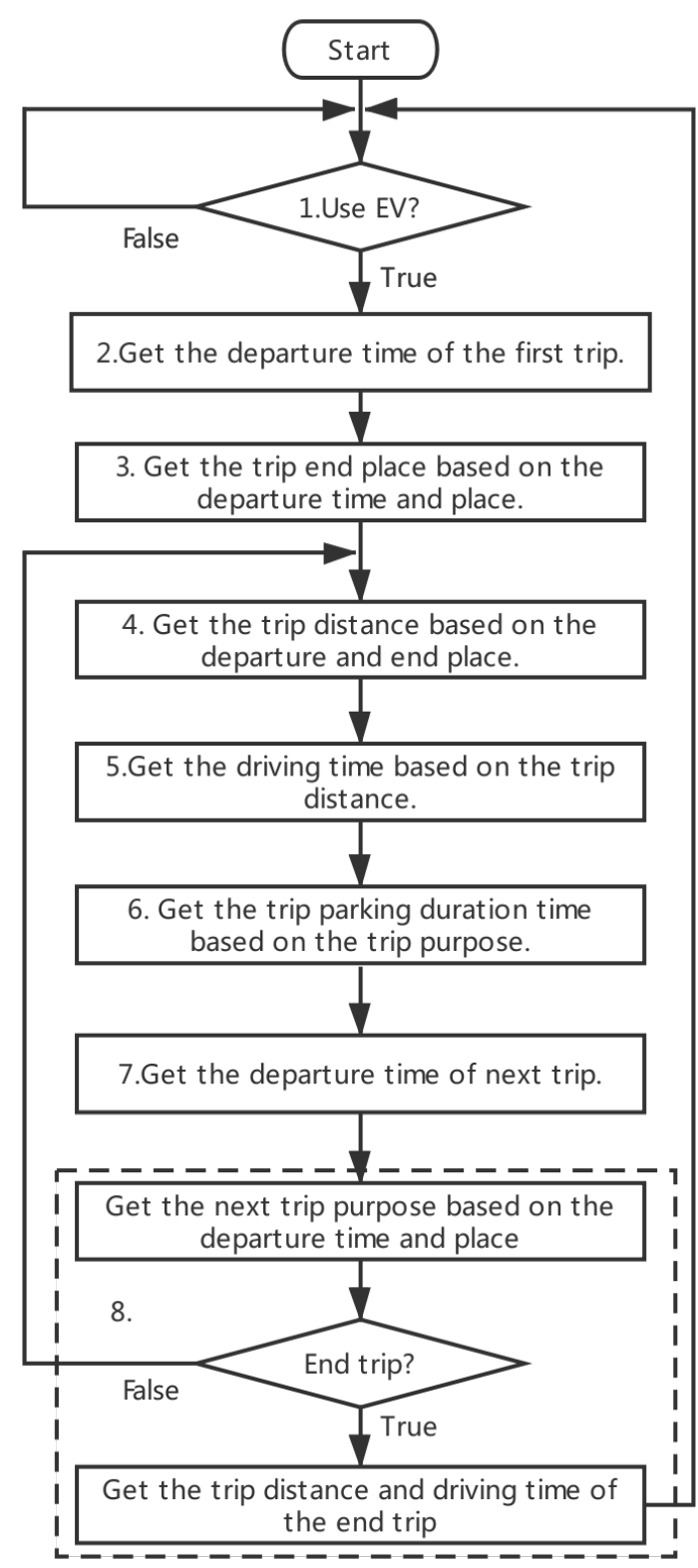

Fig.3 Trip chain generation process

The trip chain generation process is shown in Fig. 3 and it includes eight steps:

(1) If the EV is not used in a whole day, the model will not generate a trip for it and the following process will not be implemented. The probability that an EV will not be used in the day is $10.8 \%$ on weekdays and is $16.9 \%$ on weekends [30].

(2) If the EV is used, the departure time of the first trip is decided according to the PDF in Fig. 2.

(3) In the meantime, the trip purpose is determined according to the transfer probability matrix (TPM) regarding departure time and former trip purpose. The time interval for TPM extraction is one hour with NHTS dataset, at each hour, the TPM consists of six independent PDFs indicating the probability of trip purposes transformation. Detailed information of the TPMs and associated 288 PDFs are reported in supplementary data.

(4) The trip driving distance $\left(d_{t}\right)$ is then calculated according to the trip purpose with associated PDFs. The PDFs of driving distance obey the lognormal distribution, which have been 
validated using the data in the 2017 NHTS [30]. An example of $d_{t}$ between residential locations and work locations is shown in Fig.4. The details of the 36 PDFs of $d_{t}$ are provided in the supplementary data.

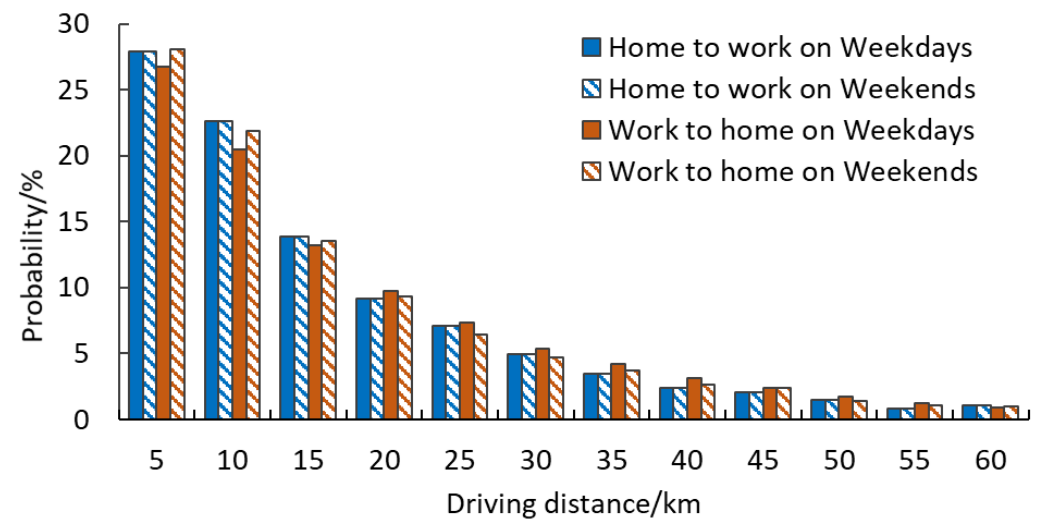

Fig.4 Distribution of driving distance between L1 and L2.

(5) The trip driving time is calculated according to the driving distance. Study [31] suggested that the driving time obeys the normal distribution of driving distance, as indicated by Formula 4, where $\mu_{t}\left(d_{t}\right)$ and $\sigma_{t}{ }^{2}\left(d_{t}\right)$ are fitted to the 2017 NHTS data [30] using power function. The PDF of trip driving time to driving distance is calculated as Formula 5.

$t_{d}=N\left(\mu_{t}\left(d_{t}\right), \sigma_{t}^{2}\left(d_{t}\right)\right)$

$\mathrm{p}\left(t_{d} \mid d_{t}\right)=\frac{1}{2.338 \cdot \sqrt{2 \pi} \cdot t_{d}^{0.5416}} e^{-\frac{1}{2 \cdot\left(2.338 \cdot t_{d}^{0.5416}\right)^{2}} \cdot\left(d-2.491 t_{d}^{0.7579}\right)^{2}}$

(6) Step (2)-(5) define a trip and the EV will park at the previous destination at the beginning of this step. The parking time $\left(t_{p}\right)$ is derived and the PDFs of $t_{p}$ vary with the following trip purpose and with the time in the day. The average parking time at the working location is shown in Fig. 5. For example, the cars arrive at four in the morning at the working location will only stay 30 minutes on average and those arrive at six will park over 500 minutes. $t_{p}$ for "home linger" purpose is the time for staying at home and the overnight parking time is the period between the ending time at home on one day and the departure time on the next day.

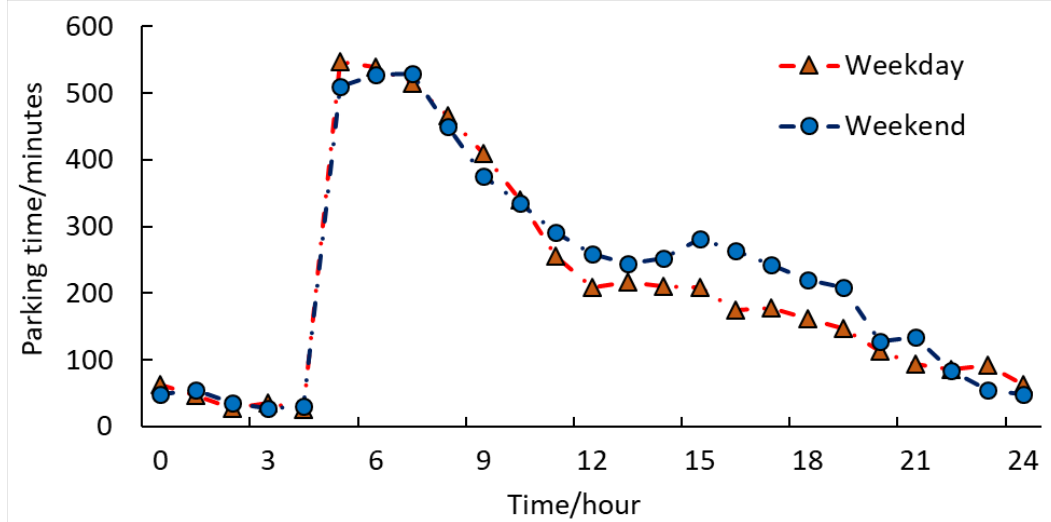

Fig.5 Average parking time at the work location

(7) The next departure time is calculated according to Formula 1 and the departure place is the destination of the last trip.

(8) The first stage of this step is similar to step (2), which obtains the trip purpose and destination. The present trip will be the last one, if the next trip purpose is "end trip". 
Otherwise, the trip generation will repeat from step (3).

\subsubsection{Validation}

Based on the distributions of four input parameters: departure time, TPM, driving distance and parking time, the key output parameters of the trip chain model: proportion of trip purposes, end trip time and number of daily trips, are examined to validate the model. To this purpose, a total of 1000 EVs were simulated for 70 days, i.e. 50 weekdays and 10 weekends ( 20 days), with a step of 1 minute. A total of 61087 trip chains including 246334 trips have been generated.

The evaluation of trip purposes between the simulation results of the trip chain model and the real-world data is shown in Table 2. The simulations regarding the seven trip purposes reflects the real-world data very well, as implied by the relative errors. Since only seven trip purposes were considered in the trip chain model, the proportions of the seven trip purposes are slightly larger than those of the real-world data. Compared to a previous study [28], in which the relative error of trip purpose was $15 \%$, the quality of the trip chain model in this study is significantly better.

Table 2. Evaluation on simulated trip purposes

\begin{tabular}{llllllllll}
\hline Purpose & P1 & P2 & P3 & P4 & P5 & P6 & P7 & Other & Total \\
\hline Real-world data (\%) & 11.01 & 14.04 & 21.14 & 7.27 & 7.78 & 10.91 & 24.44 & 3.41 & 96.7 \\
Simulation (\%) & 11.23 & 14.88 & 22.2 & 7.52 & 7.92 & 11.41 & 24.83 & 0 & 100 \\
Relative error (\%) & 1.9 & 5.8 & 5.0 & 3.4 & 1.8 & 4.6 & 1.6 & $/$ & $/$ \\
\hline
\end{tabular}

As regards time of end trip and number of daily trips, root-mean-square error (RMSE), which represents the average deviation of the simulation results from the real-world data, and correlation coefficient (CC), which represents the fitting of the simulation curve to the real-world data, were computed for validation. The results shown in Fig. 6 and Fig. 7 are much more precise than the previous method [32], which further validates that the trip chain model can accurately simulate the trip patterns of EVs.

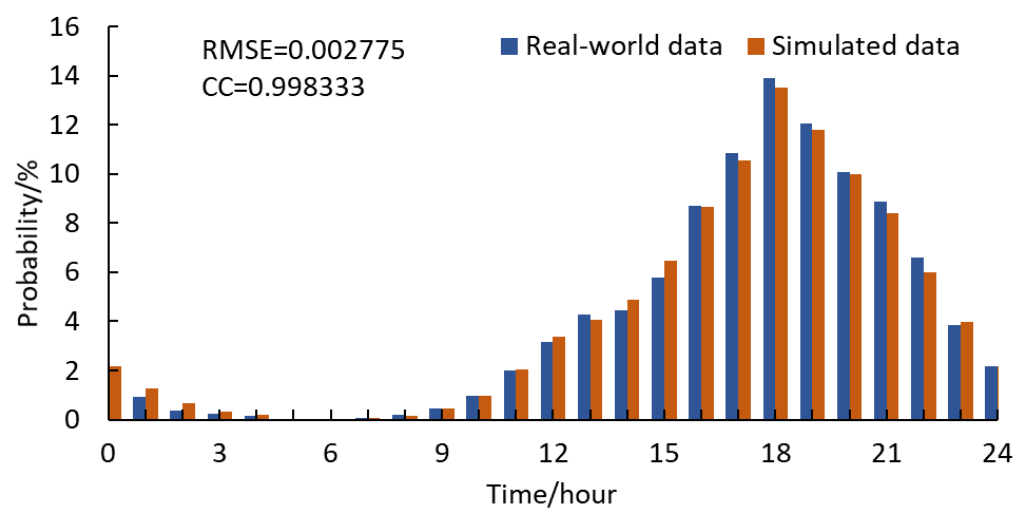

Fig.6 Distribution of the end trip time. 


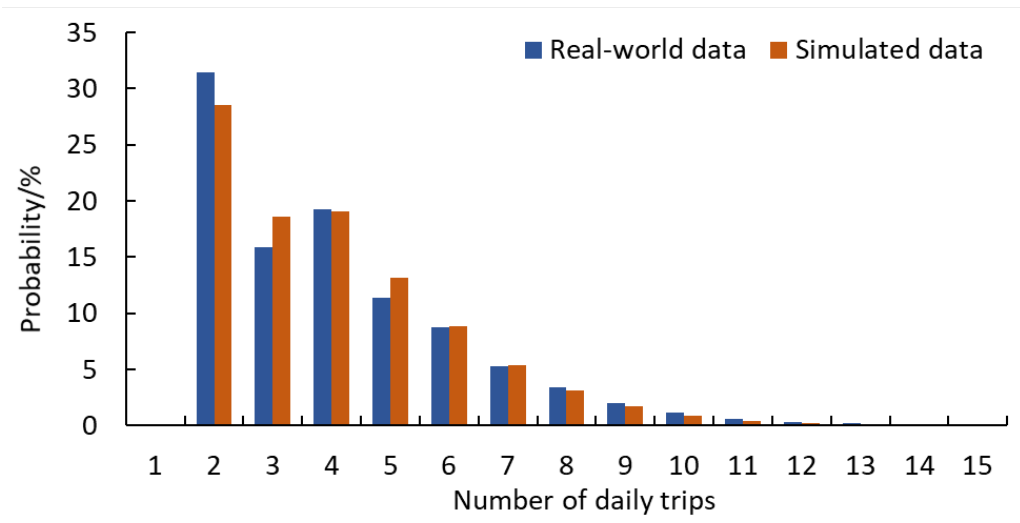

Fig.7 Distribution of the number of daily trips.

\subsection{Charging model}

The charging model simulates the charging behaviors of EVs at various locations and the charging profiles concerns about various types of EVSE and charging strategies. It is noted that the electric consumption and charging power of EV batteries are a function of many factors during the dynamic process in the real world. However, it is assumed to be a constant in the present model, because this study focuses on the generic energy performance of EVs.

\subsubsection{Electric vehicle supply equipment}

There are mainly three levels of EVSE deployed in practice: Level 1 ( $3.3 \mathrm{~kW}$ ), Level 2 (up to 14.4 kW but typically $6.6 \mathrm{~kW}$ ) and Level 3 (typically 44/120 kW) [33]. In many studies, Level 1 and Level 2 EVSE are usually referred to normal/slow charging facilities and Level 3 EVSE are called rapid/fast charging facilities. The deployment of various types of EVSE at different locations can be seen in Table 3.

Table 3. EVSE outputs and arrangement

\begin{tabular}{ccccc}
\hline EVSE & Output (kW) & Residential & Work & Public \\
\hline Level 1 & 3.3 & $\sqrt{ }$ & & \\
Level 2 & 6.6 & & $\sqrt{ }$ & $\sqrt{ }$ \\
Level 3 & 44 & & & $\sqrt{ }$ \\
\hline
\end{tabular}

\subsubsection{Charging strategy}

The movement of an EV relies on its battery, of which the power is measured by the state-ofcharge (SOC). This study assumed that the SOC of an EV is proportional to its driving distance, as shown by Formula 6 .

$\mathrm{SOC}=\mathrm{SOC}_{0}-\mathrm{d}_{t, n} \cdot \mathrm{E} / \mathrm{C}$

where $\mathrm{SOC}_{0}$ is the original SOC at the departure, $d_{t, n}$ is the trip distance, $E$ is the electricity consumption per kilometer and $\mathrm{C}$ is the battery capacity. This study considered two charging strategies: uncontrolled charging (UC) and off-peak charging (OPC) strategy, and the details are introduced as follows.

(1) UC strategy

EVs are charged according to their SOC and driving needs, while the electricity tariff or load management of the grid does not affect the charging behavior. The algorithm of the UC strategy and the detailed processes are introduced in Appendix. A1.

(2) OPC strategy

The time-of-use (TOU) pricing mechanism is employed to motivate EVs to reduce cost by 
load shifting. The OPC strategy is applied to Level 1 EVSE at home to manage EV's charging demands. A full random dispatch algorithm, which has been introduced in a previous study [27], is adopted to avoid electric imbalance. The algorithm of the OPC strategy is shown in Appendix. A2.

\subsection{Flexibility and uncertainty estimation}

\subsubsection{Evaluation of Flexibility}

Flexibility refers to the ability of adjustment of an energy system [34]. This study implemented a benchmark assessment of the charging and discharging flexibility provided by a certain EV fleet. Like other studies about flexibility, this paper utilized the maximum adjustable charging/discharging power at time $\mathrm{t}$ as ramping constraint and the maximum amount of adjustable charging/discharging electricity during the parking period as the total quantity constraint. The baseline for flexibility estimation is the original charging profile of EVs.

The maximum adjustable charging power, $P_{c h a r g e}^{\max }$, at time $\mathrm{t}$ can be calculated as Formula 7 , where $P_{\text {charger }}$ is the power of the EVSE, $m$ represents the total number of EVs and $i$ represents the ith EV. Formula (8)-(10) are the constraints for calculating the charging potential, where $t_{p, i}$ and $S O C_{i}$ represent the parking time and $\mathrm{SOC}$ of the ith $\mathrm{EV}$ and $\mathrm{C}$ represents the capacity of the battery.

$$
\begin{aligned}
& P_{\text {charge }}^{\max }=P_{\text {charger }} \cdot \sum_{1}^{m} \alpha_{t p, i} \cdot \alpha_{\text {soc }, i} \cdot \alpha_{\text {state }, i} \quad i \in\{1 \ldots m\} \\
& \alpha_{t p, i}=\left\{\begin{array}{lr}
1 & t_{p, i}>30 \mathrm{~min} \\
0 & \text { otherwise }
\end{array} \quad i \in\{1 \ldots m\}\right. \\
& \alpha_{\text {soc }, i}=\left\{\begin{array}{rr}
1 & \left(100-S O C_{i}\right) \cdot C / P_{\text {charger }}>30 \mathrm{~min} \\
0 & \text { otherwise }
\end{array} \quad i \in\{1 \ldots m\}\right. \\
& \alpha_{\text {state }, i}=\left\{\begin{array}{lr}
1 & \text { Parking } \cap \text { notCharging } \\
0 & \text { otherwise }
\end{array} \quad i \in\{1 \ldots m\}\right.
\end{aligned}
$$

Constraint (8) and (9) dictates that the adjustable charging power is counted when the parking time and the expected charging time of the ith $\mathrm{EV}$ are both longer than 30 minutes. The limitation of 30 minutes is commonly adopted by studies on smart charging or V2G activities. Constraint (10) controls the flexibility of EVs: if an EV is charging or on a trip, the adjustable charging power is zero.

The maximum amount of adjustable charging power, $C_{c h a r g e}^{\max }$, is calculated as Formula (11) and (12), where $S O C_{\max , i}$ is the maximum $\mathrm{SOC}$ that the ith $\mathrm{EV}$ can be charged during the parking time. As shown by Formula (13) and (14), it is then converted into an integral over a certain period of time for handling the sum of adjustable power over different time scales, where $P_{\text {charge, }}$ is the equivalent charging power at time t. Formula (15) is the time-related constraint for calculating $P_{\text {charge }, t}$, where $t_{d, i}$ represents the departure time of the ith EV.

$$
\begin{aligned}
& C_{\text {charge }}^{\max }=C \cdot \sum_{1}^{m}\left(S O C_{\max , i}-S O C_{i}\right) \cdot \alpha_{t p, i} \cdot \alpha_{\text {soc }, i} \cdot \alpha_{\text {state }, i} \quad i \in\{1 \ldots m\} \\
& S O C_{\text {max }, i}=\min \left(100 \%, P_{\text {charger }} \cdot t_{p, i} / C+S O C_{i}\right) \quad i \in\{1 \ldots m\} \\
& C_{\text {charge }}^{\max }=\int_{0}^{24} P_{\text {charge }, t} \cdot d t \\
& P_{\text {charge }, t}=C \cdot \sum_{1}^{m}\left(S O C_{\text {max }, i}-S O C_{i}\right) / t_{p, i} \cdot \alpha_{t p, i} \cdot \alpha_{s o c, i} \cdot \alpha_{s t a t e, i} \cdot \alpha_{t d, i} \quad i \in\{1 \ldots m\} \\
& \alpha_{t d, i}=\left\{\begin{array}{cc}
1 & t_{d, i}-t_{p, i}<t<t_{d, i} \\
0 & \text { otherwise }
\end{array} \quad i \in\{1 \ldots m\}\right.
\end{aligned}
$$

The flexibility of $V 2 G$ is calculated by a similar process as that for calculating the charging demand. The maximum adjustable V2G power and V2G amount is calculated as Formula (16)-(19), 
where $S O C_{\text {rest }, i}$ represents the necessary energy consumption of the rest trips in the day, $S O C_{a n x, i}$ represents the range anxiety of the ith $\mathrm{EV}$ driver. $V 2 G_{\max , i}$ is the maximum SOC that the ith $\mathrm{EV}$ can discharge to the grid during the parking time and the $\mathrm{SOC}$ is not allowed to go lower than $20 \%$ of the battery's full capacity for the protection purpose.

$$
\begin{aligned}
& P_{V 2 G}^{\max }=P_{V 2 G} \cdot \sum_{1}^{m} \alpha_{t p, i} \cdot \alpha_{V 2 G, i} \cdot \alpha_{\text {state }, i} \quad i \in\{1 \ldots m\} \\
& \alpha_{V 2 G, i}=\left\{\begin{array}{rr}
1 & \left(S O C_{i}-S O C_{\text {rest }, i}-S O C_{a n x, i}-20 \%\right) \cdot C / P_{V 2 G}>30 \text { min } \\
0 & \text { otherwise }
\end{array} \quad i \in\{1 \ldots m\}\right. \\
& V 2 G_{\max , i}=\min \left(S_{O C C_{i}}-S O C_{\text {rest }, i}-S O C_{a n x, i}-20 \%, P_{V 2 G} \cdot t_{p, i} / C\right) \quad i \in\{1 \ldots m\} \\
& C_{V 2 G}^{\max }=C \cdot \sum_{1}^{m} V 2 G_{\max , i} \cdot \alpha_{t p, i} \cdot \alpha_{V 2 G, i} \cdot \alpha_{\text {state }, i} \\
& =C \cdot \int_{0}^{24}\left(\sum_{1}^{m} V 2 G_{\max , i} \cdot \alpha_{t p, i} \cdot \alpha_{V 2 G, i} \cdot \alpha_{s t a t e, i} \cdot \alpha_{t d, i} / t_{p, i}\right) \cdot d t \quad i \in\{1 \ldots m\}
\end{aligned}
$$

\subsubsection{Evaluation of Uncertainty}

This study developed an evaluation parameter, namely average root-mean-square error (ARMSE), to quantify the uncertainty of charging demand. RMSE represents the average deviation of an input array from the reference array, as shown in Formula (21). Here, the input array, $X_{k, j}$, is the real-time charging demand on the $k_{\text {th }}$ day and the reference array, $\bar{X}_{j}$, is the expected average charging demand in a specific period, which is calculated as Formula (20). The ARMSE is the average value of the RMSE of $\mathrm{N}$ days, which represents the average deviation of real-time charging demand from average daily/hourly charging profile in the period of $\mathrm{N}$ days (Formula 22).

$$
\begin{array}{ll}
\bar{X}_{j}=\frac{X_{1, j}+X_{2, j}+\cdots+X_{N, j}}{N} & j \in\{1 \ldots M\} \\
R M S E_{k}=\sqrt{\frac{\sum_{j=1}^{n}\left(X_{k, j}-\bar{X}_{j}\right)^{2}}{M}} & k \in\{1 \ldots N\} \\
A R M S E=\frac{R M S E_{1}+R M S E_{2}+\cdots+R M S E_{N}}{N} &
\end{array}
$$

\section{Results}

1000 EVAs, representing $1000 \mathrm{EVs}$, were simulated in the ABTCM for 77 days, including 55 weekdays and 11 weekends ( 22 days), with a step of 1 minute. The initial SOC of each EV was assumed to be $100 \%$. The EVs were simulated for the first 7 days to enter into a random state and the simulation for the next 70 days were used to generate results. The basic information of the EVs and the details of the TOU pricing mechanism [35] are provided in Appendix.A3.

\subsection{Distribution of charging demand}

\subsubsection{Charging profiles of EVs under the uncontrolled charging strategy}

Table 4 shows the average daily charging demand of $1000 \mathrm{EV}$ s under the UC strategy. In general, about $50 \%$ of the total charging demand (TCD) is met at the home location, followed by the work location (about 21\%) and public location (about 29\%). In addition, the daily charging demand on weekdays on average is $17 \%$ higher than that on weekends. The most significant difference occurs at the work location, i.e. the charging demand at work locations (WCD) on a weekday is nearly 5 times of that on a weekend. By contrast, the charging demand at public locations (PCD) becomes larger on weekends, especially the demand associated with recreation purpose (RCD) on a weekend nearly doubles the amount on a weekday. 
Table 4. Average daily charging demand of 1000 EVs under UC strategy

\begin{tabular}{|c|c|c|c|c|c|c|c|c|c|}
\hline \multirow{3}{*}{$\begin{array}{l}\text { Location } \\
\text { EVSE/kWh }\end{array}$} & \multirow{3}{*}{$\begin{array}{l}\text { Home } \\
\text { Slow }\end{array}$} & \multirow{3}{*}{$\begin{array}{l}\text { Work } \\
\text { Slow }\end{array}$} & \multicolumn{6}{|c|}{ Public } & \multirow{3}{*}{$\begin{array}{c}\text { Total } \\
/\end{array}$} \\
\hline & & & \multicolumn{2}{|c|}{ Shopping } & \multicolumn{2}{|c|}{ Meal } & \multicolumn{2}{|c|}{ Recreation } & \\
\hline & & & Slow & Fast & Slow & Fast & Slow & Fast & \\
\hline Weekday & 5019.5 & 2601.3 & 620.5 & 350.0 & 341.3 & 125.3 & 937.1 & 171.3 & 10166.2 \\
\hline Weekend & 4544.5 & 564.5 & 704.8 & 331.9 & 466.7 & 164.1 & 1665.7 & 270.5 & 8712.5 \\
\hline
\end{tabular}

The average daily charging profiles on weekdays and weekends under the UC strategy are illustrated in Fig. 8. The patterns of the charging demand at the home location (HCD) and WCD are unimodal, with the peaks appearing at around 20:00 and at 9:00, respectively. Due to the peaks of the HCD and WCD, the pattern of the TCD is bimodal on weekdays and is unimodal on weekends. The profiles of PCD are much more fluctuant than those of the HCD and WCD due to the utilization of level 3 EVSE. On weekends, the average peak of PCD occurring at around 11:00 am is larger than that of HCD. This study considered three purposes, namely meal, shopping and recreation, at the public location and these represent the general characteristics of PCD. When a public location provides only one function or other functions, the PCD will change accordingly.

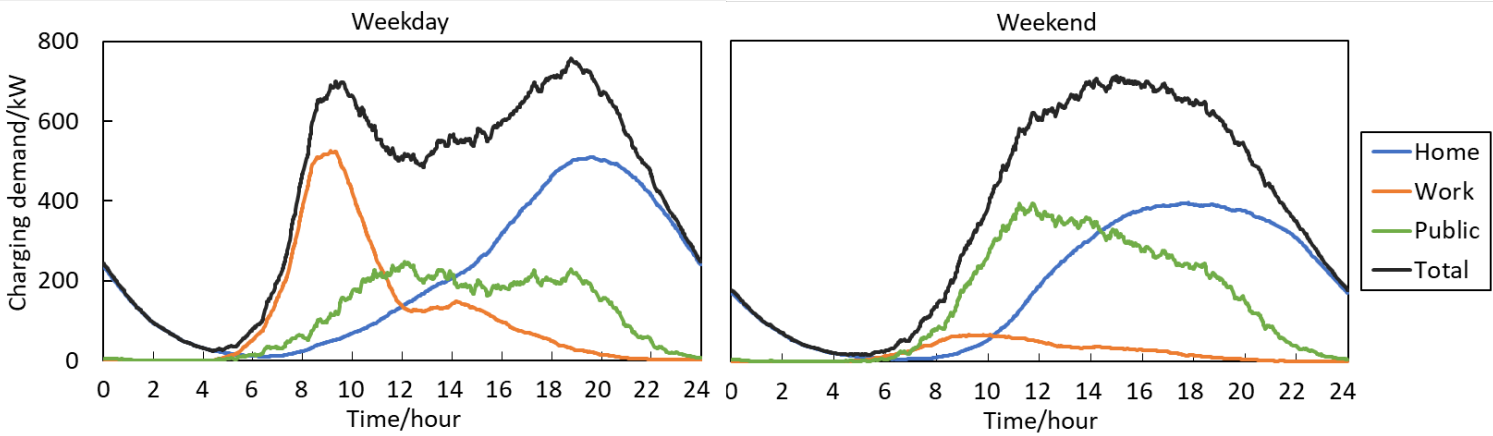

Fig.8 Average daily charging profiles under uncontrolled charging strategy.

Shown in Fig. 9, the patterns of charging demand for shopping purpose (SCD) and meal purpose (MCD) do not change much on different days, while the increase in recreation activities on weekends significant stimulate the RCD, as well as the PCD.

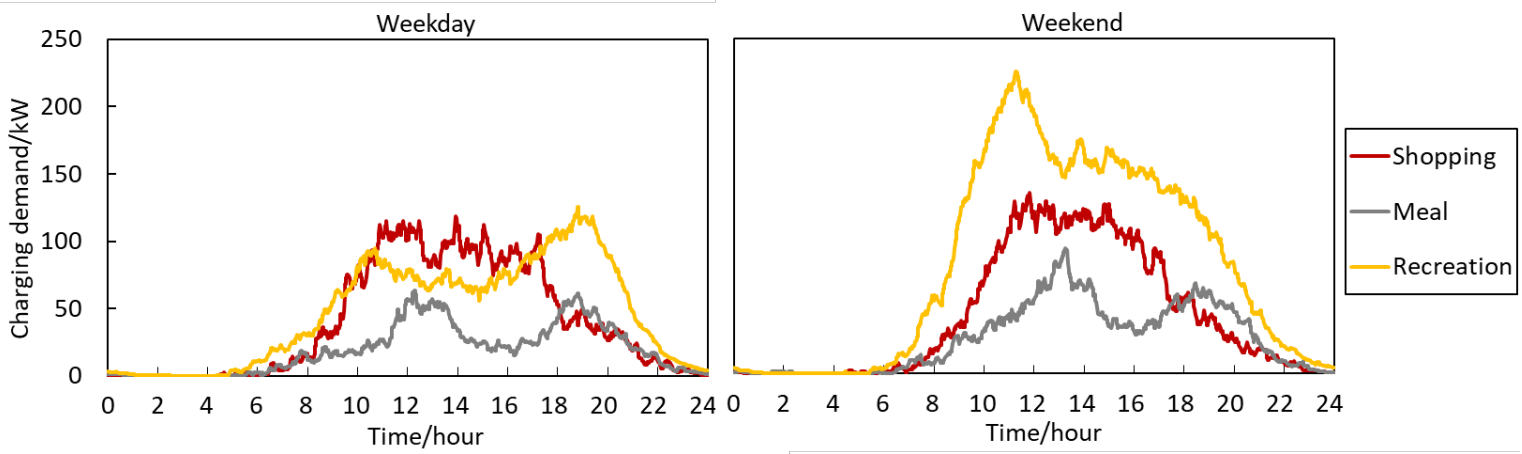

Fig.9 Average daily charging profiles at public locations under uncontrolled charging strategy.

\subsubsection{Charging profiles of EVs under the off-peak charging strategy}

The average daily charging demand of EVs under the OPC strategy is shown in Table 5. Compared to the demand under the UC strategy, the HCD is much larger, representing nearly $75 \%$ 
of the TCD. The PCD accounts for about $15 \%$ of the TCD and the WCD accounts approximately $10 \%$ of the TCD.

Table 5. Average daily charging demand of 1000 EVs under OPC strategy

\begin{tabular}{|c|c|c|c|c|c|c|c|c|c|}
\hline \multirow{3}{*}{$\begin{array}{l}\text { Location } \\
\text { EVSE/kWh }\end{array}$} & \multirow{3}{*}{$\begin{array}{c}\text { Home } \\
\text { Slow }\end{array}$} & \multirow{3}{*}{$\begin{array}{l}\text { Work } \\
\text { Slow }\end{array}$} & \multicolumn{6}{|c|}{ Public } & \multirow{3}{*}{$\begin{array}{c}\text { Total } \\
\text { / }\end{array}$} \\
\hline & & & \multicolumn{2}{|c|}{ Shopping } & \multicolumn{2}{|c|}{ Meal } & \multicolumn{2}{|c|}{ Recreation } & \\
\hline & & & Slow & Fast & Slow & Fast & Slow & Fast & \\
\hline Weekday & 7507.5 & 1226.0 & 336.8 & 182.7 & 203.6 & 77.1 & 587.0 & 92.2 & 10212.8 \\
\hline Weekend & 6615.5 & 275.0 & 336.6 & 175.7 & 216.7 & 79.5 & 844.3 & 136.4 & 8700.4 \\
\hline
\end{tabular}

The average charging profiles of EVs under the OPC strategy are illustrated in Fig. 10. Different from the original bimodal TCD under the UC strategy, the TCD under the OPC strategy becomes a unimodal pattern and the peak load appears in the period of 23:00-7:00 (day+1). The average peak load under the OPC strategy rises up to $1383 \mathrm{~kW}$ on weekdays and $1195 \mathrm{~kW}$ on weekends, representing an $83 \%$ and a $68 \%$ increase over the peak loads under the UC strategy. This is mainly attributed to the changes in the HCD, i.e. the peaks move to the period of 23:00-7:00 (day+1) and increase by $171 \%$ on weekdays and by $201 \%$ on weekends (Fig. 10 cf. Fig. 8 ). In the meantime, the WCD and PCD both significantly decrease.

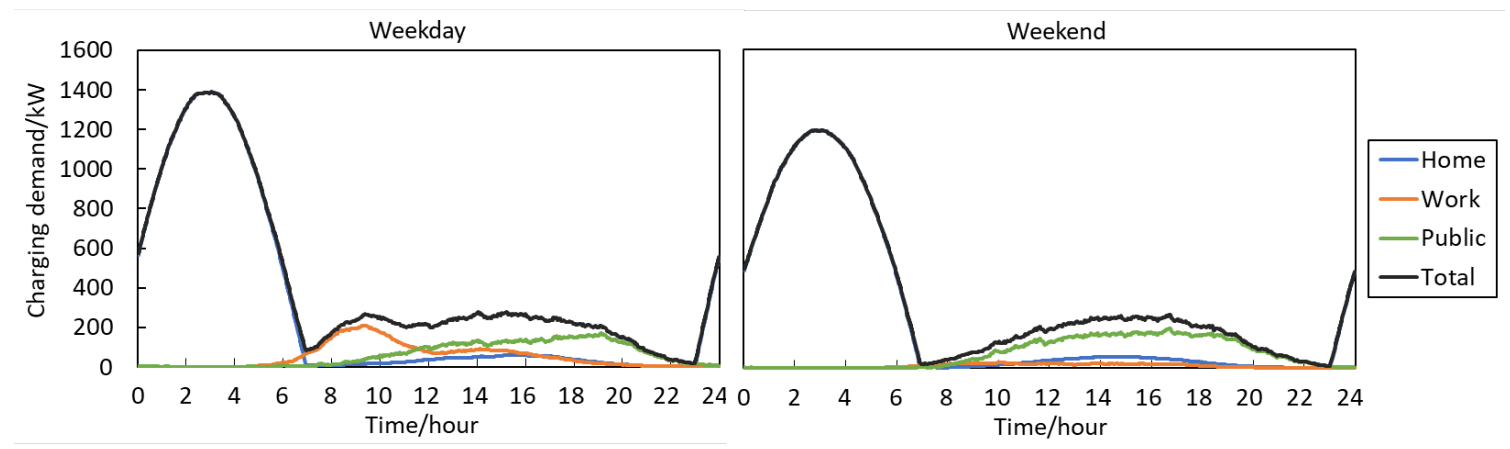

Fig.10 Average daily charging profiles under off-peak charging strategy.

The average daily charging profiles at public locations under the OPC strategy are shown in Fig.11. With the increase in HCD, as indicated in Fig. 11, the average PCD significantly decreases, especially the peak load of RCD in the morning vanishes (Fig. 11 cf. Fig. 9).

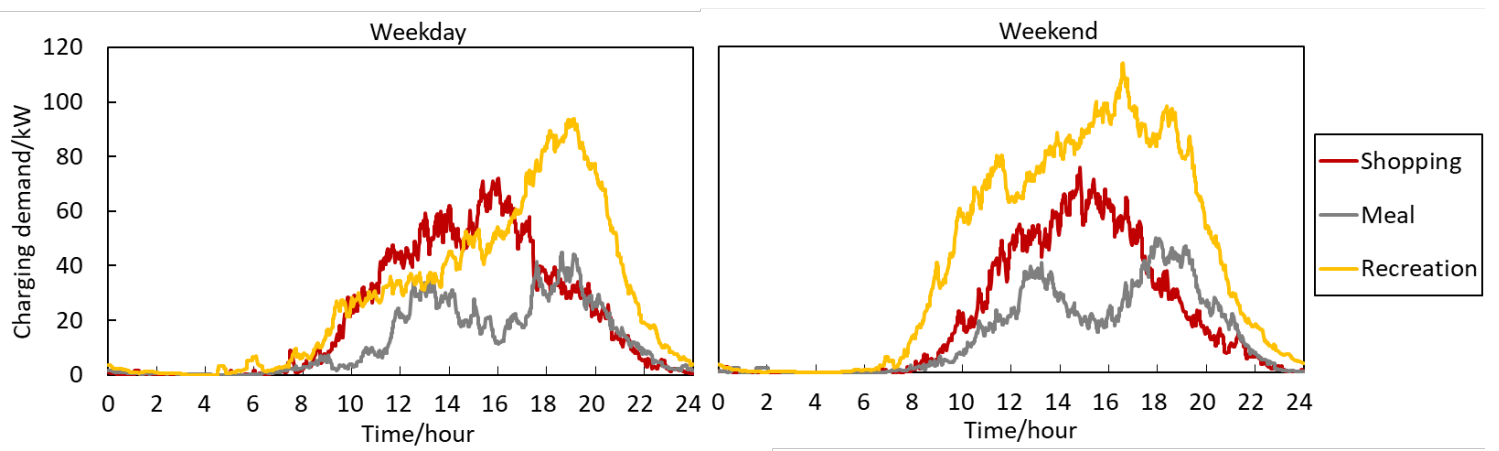

Fig.11 Average daily charging profiles at public locations under off-peak charging strategy.

As a result of load shifting, the daily electricity cost under the OPC strategy is reduced by about $40 \%$ compared to that under the UC strategy under the TOU pricing mechanism (Fig. 12). 


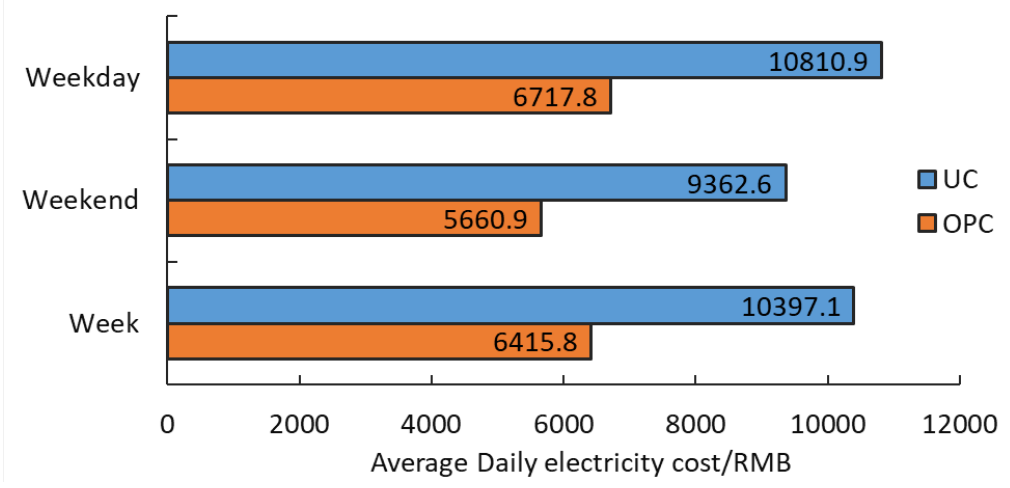

Fig.12 Average daily electricity cost under different charging strategies

\subsubsection{The influence of traffic flow on charging demand}

Traffic flow, represented by number of parking EVs, can be easily acquire in practice. Traffic flow can be used to forecast charging demand, which is the most important information for planning and operating a charging station. Fig. 13 shows the average daily traffic flows at different locations, where the flows at the public locations are further divided according to trip purpose. The largest aggregate traffic flow occurs at the public location and smallest at the work location. While the traffic flow at public locations increases at weekends, the traffic flow at work locations decreases more on weekends.

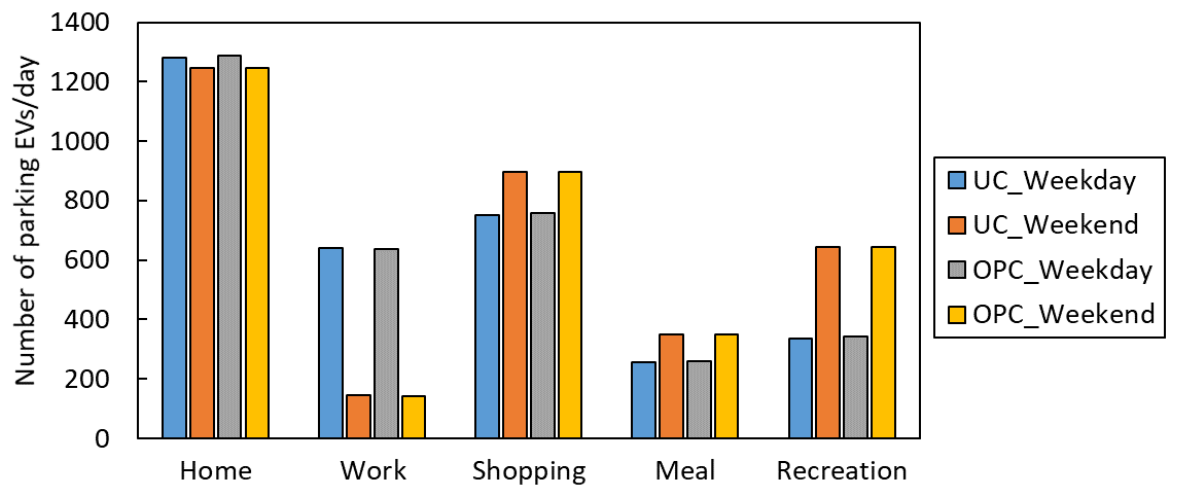

Fig. $13 \mathrm{EV}$ average daily traffic flow at different locations

To reveal the relation between traffic flow and charging demand, equivalent daily charging demand (EDCD) and equivalent daily peak load (EDPL) are calculated as Formula (23)-(24). Different from the heterogenous impacts of an individual EV, EDCD indicates the amount of charging demand contributed by one EV on average and EDPL shows the magnitude of peak load contributed by one EV on average. EDCD and EDPL reflect the synthetic effects of charging possibility, charging duration, EVSEs and discrete charging distribution. However, EDCD and EDPL are much easier to obtain and more applicable in practice than the rest parameters.

$$
\begin{aligned}
E D C D & =\frac{\text { Daily charging demand }}{\text { Traffic flow in a day }} \\
E D P L & =\frac{\text { Peak load of Daily charging profile }}{\text { Traffic flow in a day }}
\end{aligned}
$$

The distributions of the EDCD and the EDPL are shown in Fig. 14 and 15. Although there is the largest traffic flow and fast charging EVSE are available at public locations, the EDCDs and EDPLS 
are the smallest. This reveals the lowest charging demand on average at public locations, mainly because of the shortest time of charging. The EDCDs at home and work locations are quite similar under the UC strategy, while it becomes much higher at home locations under the OPC strategy, due to the load shifting effect of the TOU pricing (Fig. 14). The impact of the OPC strategy on EDPL at home locations is even larger (Fig. 15), implying that most EVs charge when electricity prices is low and that peak load shifts to another time due to this effect. The EDPLs at work locations are the largest under the UC strategy, because the charging time of different EVs overlaps. In addition, the ranges of distribution of EDCD and EDPL are widest at work locations, reflecting the largest variations in charging demand at work locations.

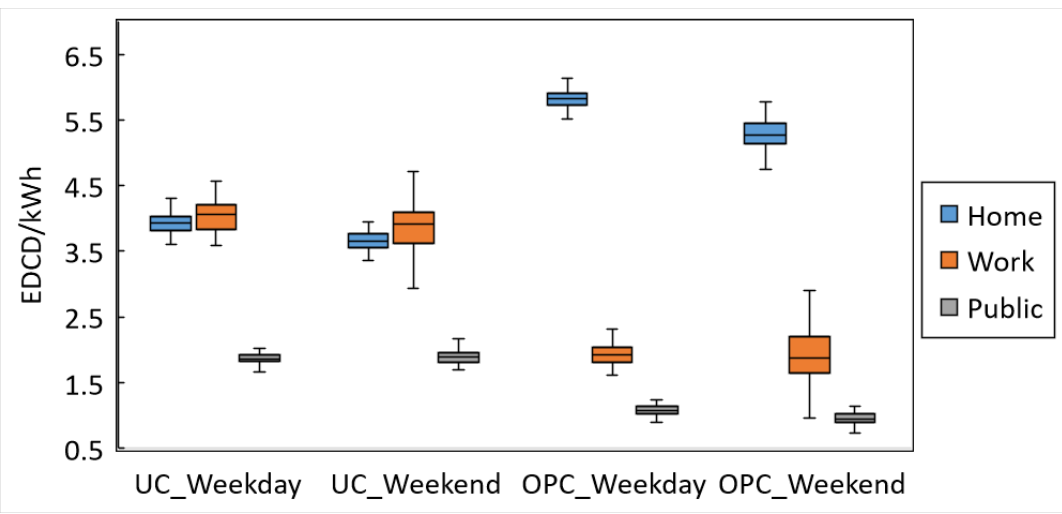

Fig. 14 The equivalent daily charging demand of EVs at different locations.

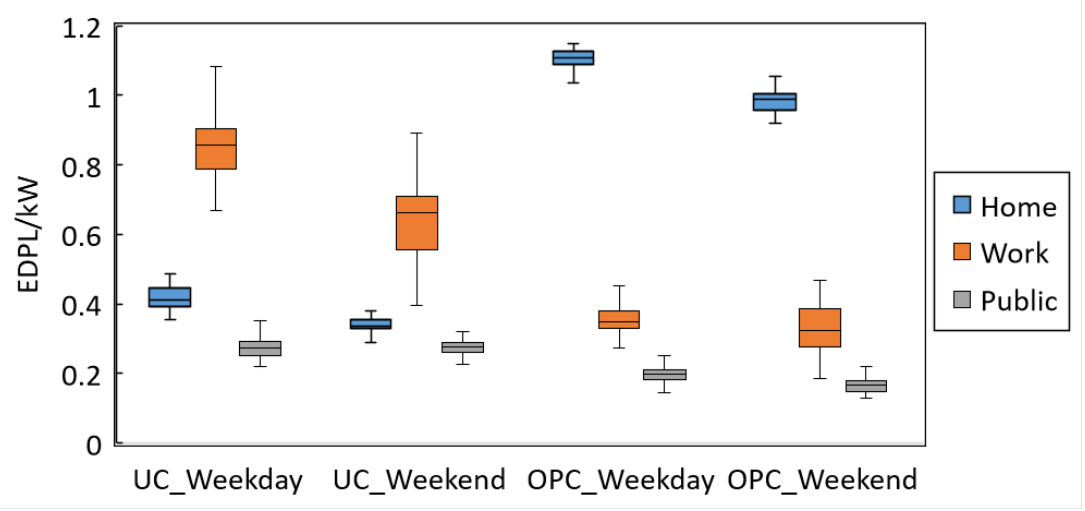

Fig. 15 The equivalent daily peak load of EVs at different locations.

The EDPL of each purpose at public locations was further calculated and the results are shown in Fig. 16. It is interesting to see that the EDPLs of individual purposes are much larger than the overall EDPL at public locations. This phenomenon indicates that the charging loads related to different activities are discretely distributed, i.e. can be called "Discrete peak effect". The discrete charging plus a short charging time leads to small EDPLs at public locations. In addition, the individual EDPLs of shopping and meal purposes are at an equivalent level of the EDPLs at home and work locations, indicating the effect of fast charging. In practice, it is important to consider the discrete peak effect in organizing the loads of EV charging and to integrate EDCDs and EDPLs into the Geographic Information System (GIS) to solve, e.g. planning for the development of EV infrastructure [36]. 


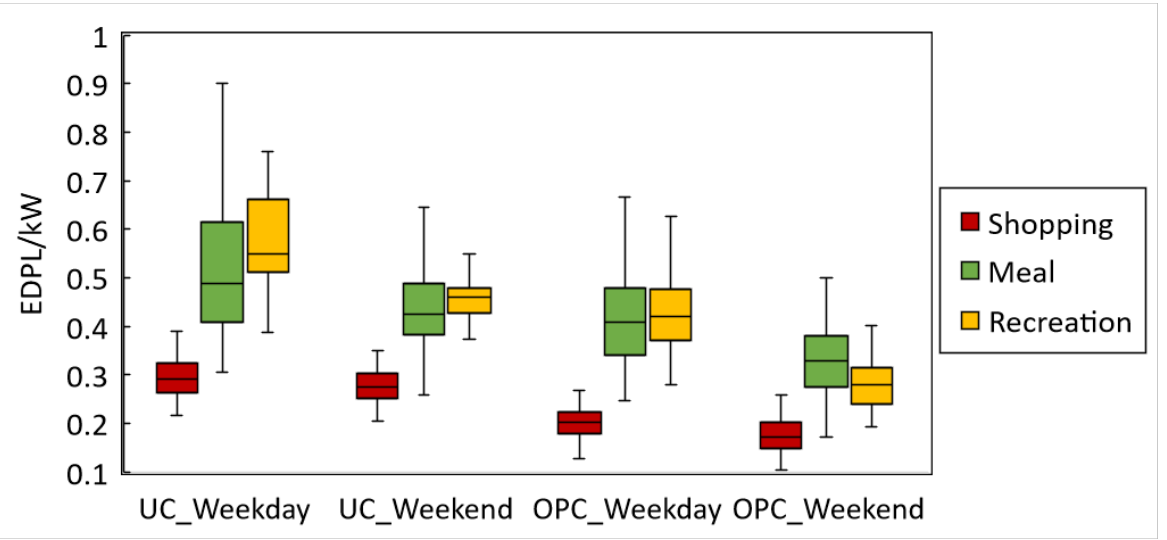

Fig. 16 The daily peak load of EVs for different purposes at public location

\subsection{Flexibility of charging and discharging of EVs}

\subsubsection{Charging flexibility of EVs}

Charging flexibility is evaluated by the maximum adjustable charging power, $P_{c h a r g e}^{\max }$, and the maximum adjustable amount of charging electricity, $C_{c h a r g e}^{\max }$, of which the results are shown in Fig.17 and Table 6, respectively. Since all of the EVs at home locations participate in load shifting, the maximum adjustable charging power, $P_{c h a r g e}^{\max }$, and the maximum adjustable amount of charging electricity, $C_{\text {charge, }}^{\text {max }}$ at home locations under the OPC strategy were not calculated.

As shown in Fig.17 and Table 6, the EVs at home locations have the largest charging flexibility, $P_{\text {charge }}^{\max }$ and $C_{\text {charge }}^{\max }$, under the UC strategy. However, in the period 9:00-15:00 on weekdays, which is the regular working time, the maximum adjustable charging power $P_{c h a r g e}^{\max }$ at home locations is smaller than that at work locations (Fig. 17 (a)).

On weekends, the charging flexibility, $P_{c \text { harge }}^{\max }$ and $C_{\text {charge, }}^{\max }$ at work locations greatly decreases due to the reduction in traffic flow and parking time. By contrast, the charging flexibility, $P_{c h a r g e}^{\max }$ and $C_{c h a r g e}^{\max }$, at public locations is higher on weekends than on weekdays due to a larger traffic flow and longer parking time (Fig. 17 (a) cf. (b) and (c) cf. (d)).

Charging strategies, i.e. the UC and OPC strategy, do not change the overall pattern of the maximum adjustable charging power, $P_{c \text { harge, }}^{\max }$, only the corresponding value of the profile have proportionally reduced (Fig. 17 (a) cf. (c) and (b) cf. (d)). The maximum adjustable amount of charging electricity, $C_{\text {charge }}^{\max }$, at work and public locations under the OPC strategy is $29 \%$ and $24 \%$ smaller than the adjustable amount under the UC strategy, since people tend to charge during the off-peak period.

Although the maximum adjustable charging power, $P_{\text {charge, }}^{\max }$ at public locations is comparatively small, the adjustable amount of electricity, $C_{c h a r g e}^{\max }$, is large enough for load shifting. This is also related to the discrete charging effect, which enables a long time for load shifting at public locations. The method proposed in this study, with case specific data, can provide valuable information on load management and grid control. 


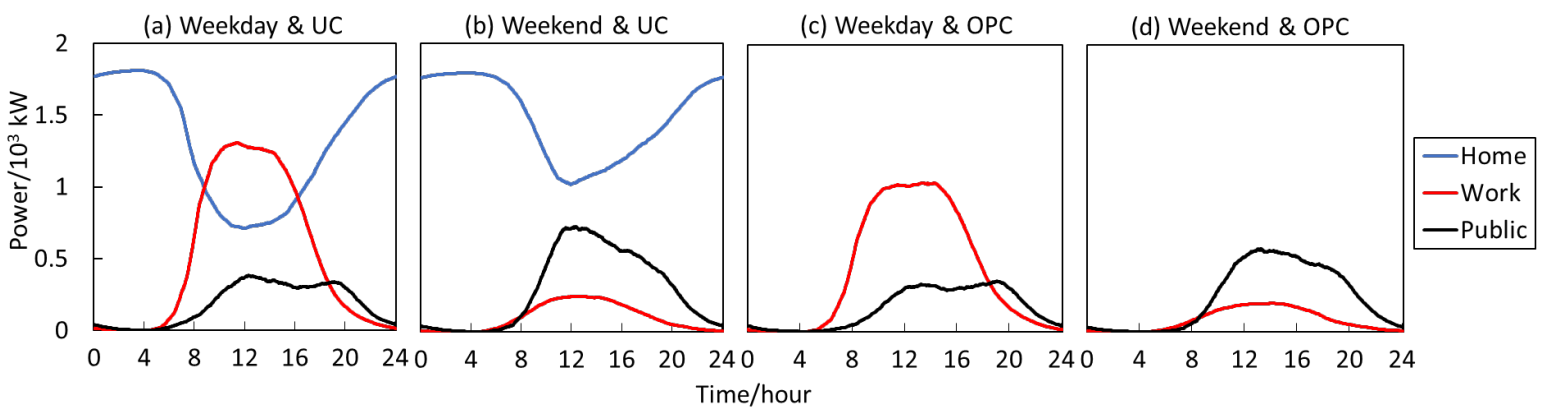

Fig. 17 The maximum adjustable charging power profiles at different locations.

Table 6. Average daily maximum adjustable amount of charging electricity (kWh)

\begin{tabular}{ccccccc}
\hline \multirow{2}{*}{ Location } & \multirow{2}{*}{ Home } & \multirow{2}{*}{ Work } & \multicolumn{4}{c}{ Public } \\
\cline { 4 - 7 } & & & Shopping & Meal & Recreation & Total \\
\hline UC_Weekday & 5316.3 & 2323.7 & 994.4 & 588.0 & 1199.8 & 2782.3 \\
UC_Weekend & 5360.0 & 527.2 & 1213.3 & 793.0 & 2318.2 & 4324.5 \\
OPC_Weekday & $/$ & 1651.9 & 804.5 & 488.7 & 996.5 & 2289.7 \\
OPC_Weekend & $/$ & 370.1 & 903.8 & 638.7 & 1599.3 & 3141.9 \\
\hline
\end{tabular}

\subsubsection{Flexibility of vehicle-to-grid}

V2G flexibility is evaluated by the maximum adjustable V2G power, $P_{V 2 G}^{\max }$, and the maximum adjustable amount of $\mathrm{V} 2 \mathrm{G}$ electricity, $C_{V 2 G}^{\max }$, of which the results are shown in Fig.18 and Table 7, respectively.

The maximum adjustable V2G power, $P_{V 2 G}^{\max }$ (Fig. 18), shows the same pattern as the maximum adjustable charging power, $P_{c h a r g e}^{\max }$ (Fig. 17), because they are both closely related to traffic flows.

The V2G flexibility, $P_{V 2 G}^{\max }$ and $C_{V 2 G}^{\max }$, is generally larger than charging flexibility, $P_{c \text { charge }}^{\max }$ and $C_{\text {charge }}^{\max }$, at work and public locations. On the contrary, the maximum adjustable amount of V2G electricity, $C_{V 2 G}^{\max }$, at home location is smaller than the adjustable amount of charging electricity $C_{\text {charge }}^{\max }$, which shows that for many EVs the capacity for V2G is limited due to a lower SOC after the trips in daytime. The maximum adjustable amount of $\mathrm{V} 2 \mathrm{G}$ electricity, $C_{V 2 G}^{\max }$, at public locations is larger than the amount at home and work locations, although the maximum adjustable V2G power, $P_{V 2 G}^{\max }$, is relatively small.

Charging strategy has an opposite and heavier influence on the V2G flexibility than on the charging flexibility, i.e. the V2G flexibility under the OPC strategy at work and public locations is larger than that under the UC strategy. This is because EVs usually have a larger SOC after the OPC operation at home and thus have larger potentials of discharging and smaller potentials of charging in the following period of time at work and public locations.

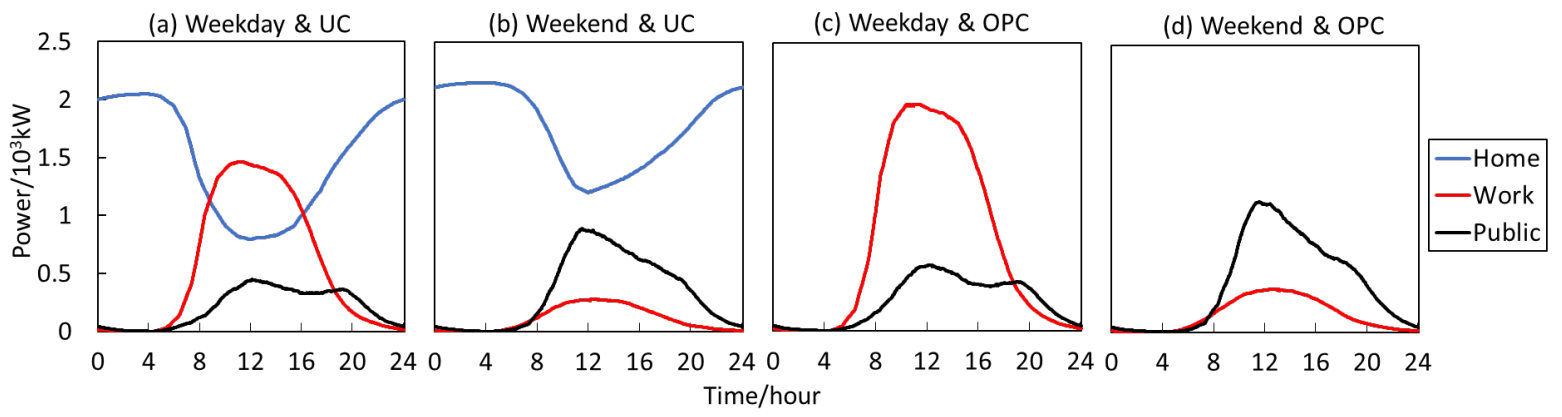

Fig. 18 The maximum adjustable V2G power profiles at different locations.

Table 7. Average maximum amount of adjustable V2G electricity (kWh) 


\begin{tabular}{ccccccc}
\hline \multirow{2}{*}{ Location } & \multirow{2}{*}{ Home } & \multirow{2}{*}{ Work } & \multicolumn{5}{c}{ Public } \\
\cline { 4 - 7 } & & & Shopping & Meal & Recreation & Total \\
\hline UC_Weekday & 4674.1 & 2732.6 & 1231.8 & 727.6 & 1338.0 & 3297.4 \\
UC_Weekend & 3233.5 & 589.6 & 1520.6 & 996.1 & 2742.7 & 5259.4 \\
OPC_Weekday & $/$ & 4161.5 & 1541.2 & 917.0 & 1880.7 & 4338.9 \\
OPC_Weekend & $/$ & 884.3 & 1898.8 & 1254.0 & 3794.6 & 6947.4 \\
\hline
\end{tabular}

\subsection{The uncertainty of charging demand}

Not only the total magnitude of EV charging demand, but also its dynamic characteristics are of great importance, because the uncertainty of $\mathrm{EV}$ charging demand can greatly affect the reliability of power systems [37]. Due to the heterogeneity and randomness in EVs' travel and charging behavior, the forecast of EV charging demand may contain a high level of uncertainty, especially with respect to fast charging. Therefore, the study calculated ARMSE to measure the uncertainty of charging demand.

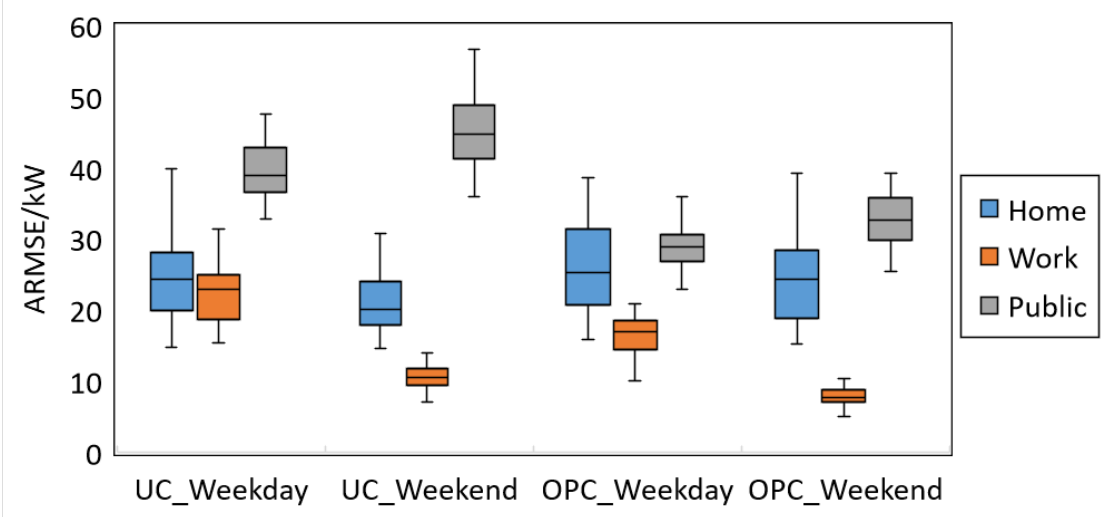

Fig. 19 The uncertainty of daily charging profile at different locations (time step: 1 minute)

Fig 19 compared the uncertainty of charging demands at different locations over a day. The uncertainties of charging demand at public locations are highest under different charging strategies, and this is due to the application of Level 3 EVSE.

Apart from the influence of EVSE, the ARMSE is positively related to charging demand. This is why the uncertainties of charging demand at home locations are generally higher than those at work locations and why the uncertainties on weekdays are higher than those on weekends. Compared to the uncertainties under the UC strategy, the ARMSEs at work and public locations under the OPC strategy are much lower, meaning meanwhile the OPC strategy motivates loadshifting, it also mitigates uncertainty of charging demand.

\section{Conclusions}

An agent-based trip chain model (ABTCM) was proposed to simulate the traffic patterns and charging demand of electric vehicles (EVs). Based on the heterogenous travel and charging patterns of EVs, not only the total magnitude of EV charging demand, but also its dynamic characteristics, including flexibility and uncertainty, at different types of location were analyzed in this study.

The largest charging demand, about $50 \%$ of the total, is met at home locations, under the uncontrolled charging (UC) strategy and about $70 \%$ of the total demand is met at home locations 
under the off-peak charging (OPC) strategy. The second largest part of charging demand is met at work locations on weekdays, but the smallest charging demand is met at work locations on weekends. Under OPC strategy, the peak loads move away from the original periods and this results in a $40 \%$ cost reduction compared to that under the UC strategy.

The relation between traffic flow and charging demand was revealed by equivalent daily charging demand (EDCD) and equivalent daily peak load (EDPL). Under the UC strategy, the EDPLS at work locations are largest, because the charging time of different EVs overlaps. Under the OPC strategy, the EDCDs and EDPLs at home locations become much larger due to load shifting. The discrete peak effect plus a short charging time leads to the smallest EDCDs and EDPLs at public locations, although there is the largest traffic flow and fast charging supply equipment is available. In practice, it is important to consider the discrete peak effect in organizing the loads of $E V$ charging and to integrate EDCDs and EDPLs into the planning for the development of EV infrastructure.

EV charging and V2G flexibility were examined by instantaneous maximum adjustable charging power and accumulative maximum adjustable amount of charging electricity. On the whole, the EVs at home locations have the largest charging and V2G flexibility under the UC strategy; except for a period of regular working time, when the maximum adjustable charging power at home locations is smaller than that at work locations. Meanwhile, the V2G flexibility at work and public locations, is generally larger than charging flexibility. In addition, the discrete peak effect causes a long time for load shifting at public locations. As a result, the adjustable amount of electricity is large at public locations, while the adjustable power is comparatively small.

Due to the application of fast charging supply equipment, the uncertainties of charging demand at public locations are the highest in all locations. Compared to the results under the UC strategy, the uncertainties at work and public locations are much lower under the OPC strategy, meaning meanwhile the OPC strategy motivates load-shifting, it also mitigates the uncertainty of charging demand.

The method proposed in this study, as well as the results, provide important information on how charging stations should be planned and operated, and on how demand-side energy management should be implemented at different types of location. Future research can incorporate the GIS information on land-use type and traffic flow into the methodology to simulate dynamic charging demand and thus to assist in planning for charging stations in the real world. In addition, the degradation of EV batteries can be considered in a detailed powertrain model to explore life-time utilization of batteries.

\section{Acknowledgements}

This work was supported by Project U1864202 supported by National Natural Science Foundation of China, the Fundamental Research Funds of Shandong University (2018JC060), China and the Gustav Dahl Scholarship, Sweden. 


\section{Appendix}

A1. Uncontrolled charging strategy

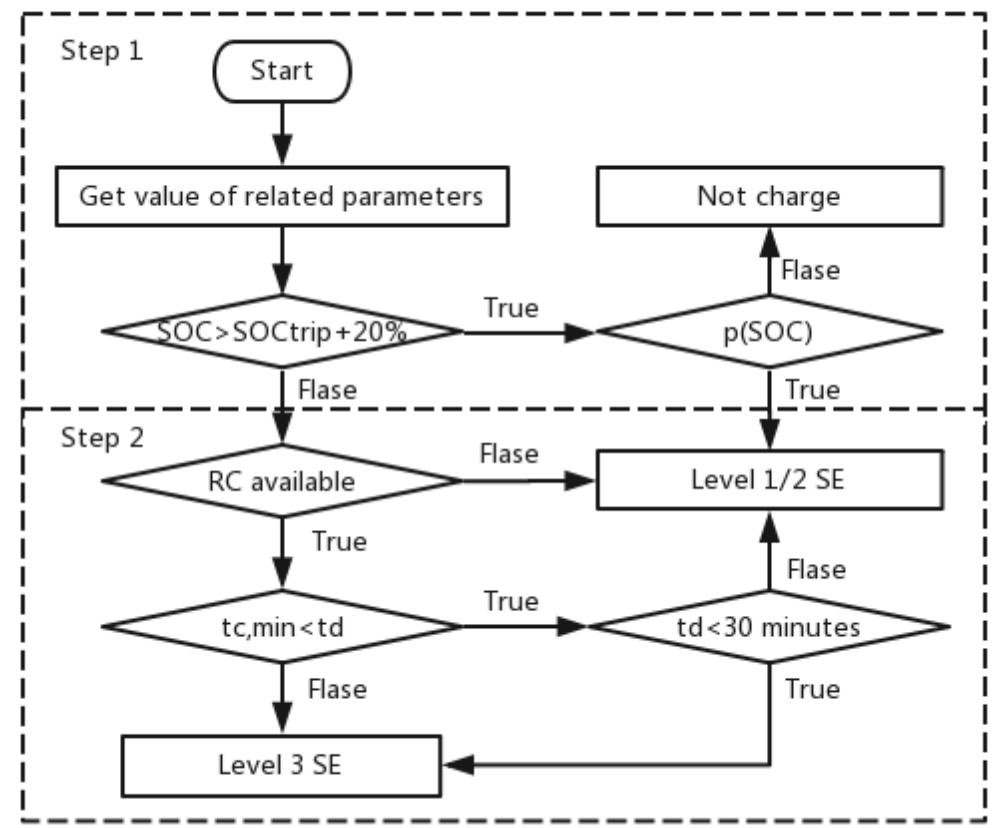

Fig.A1 Algorithm of uncontrolled charging strategy

Step 1: The probability of charging for a given state of charge (SOC), expressed by $\mathrm{p}(\mathrm{SOC})$, and the battery electricity needed for the next trip, expressed by $\mathrm{SOC}_{\text {trip, }}$, are calculated at the beginning of the process. If the current battery SOC is sufficient for the next trip (20\% SOC is designed for battery protection), the charging event is determined according to $\mathrm{p}(\mathrm{SOC})$. If $\mathrm{p}(\mathrm{SOC})$ is true, the EV will charge immediately. If $\mathrm{p}(\mathrm{SOC})$ is false, the EV will not charge. The probability density functions (PDFs) for $\mathrm{p}(\mathrm{SOC})$ at different locations and its related real-world data based SOC distribution are shown in Fig. A2. $\mathrm{SCO}_{\text {trip }}$ is calculated as Formula A1.

$S O C_{\text {trip }}=2 \times d_{t} \times E / C$

Step 2: If the SOC is not sufficient for the next trip, the EV has to charge immediately. The minimum SOC before leaving, expressed by $\mathrm{SOC}_{\mathrm{min}}$, and the corresponding charging time $t_{c, \min }$ at Level1/2 SE are calculated for the selection SE. If the time for charging the battery to its full capacity is longer than the parking time, i.e. $\mathrm{mt}_{c}>t_{d}$, or the parking time is shorter than 0.5 hour, the EV will charge at the Level 3 SE. Otherwise, the EV will charge at the Level $1 / 2 \mathrm{SE}$. The $\mathrm{SOC}_{\min }$ and $t_{c, \text { min }}$ are calculated according to Formula $\mathrm{A} 2$ and $\mathrm{A} 3$, where $\mathrm{P}$ and $\mathrm{e}$ represent the charging power and efficiency of Level 1/2 SE.

$S O C_{\min }=\min \left(100 \%, 20 \%+S O C_{\text {trip }}\right)$

$t_{c, \text { min }}=\left(S O C_{\text {min }}-S O C\right) \cdot C /(P \cdot e)$ 


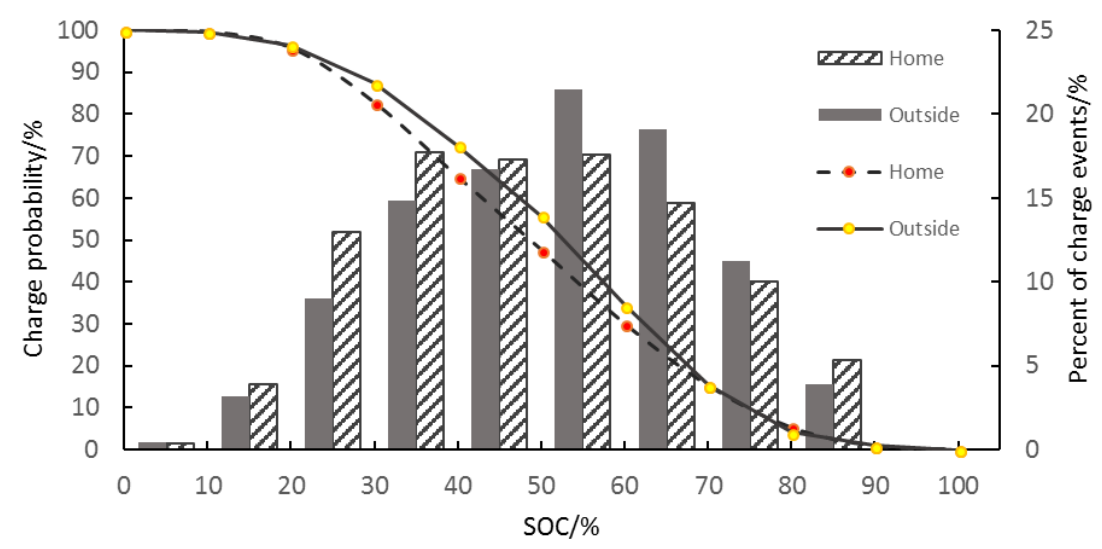

Fig.A2 PDF of $p(S O C)$ and the distribution of SOC at the start of charging events Source: calculated from [38].

A2. Off-peak charging strategy

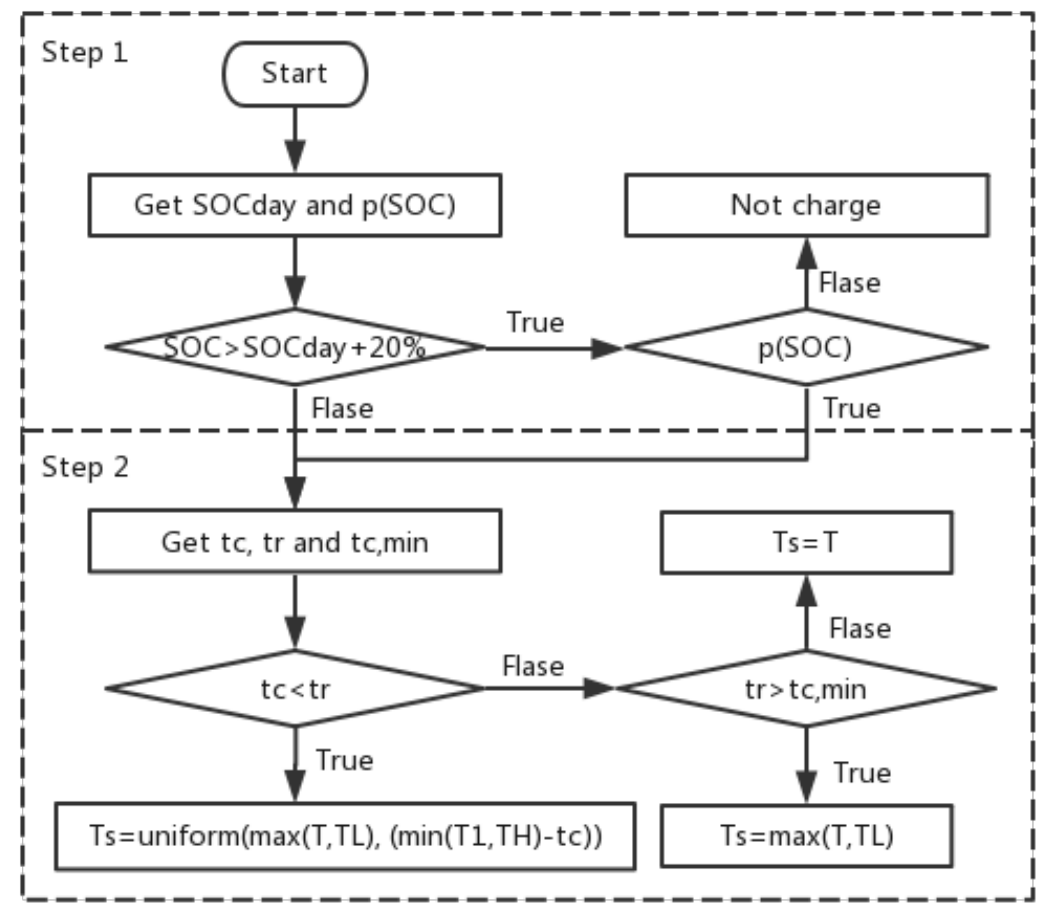

Fig.A3 Algorithm of smart charging strategy

Step 1: $\mathrm{SOC}_{\text {day }}$ represents the electricity needed for the next day's trip and it is calculated as Formula A4.

Step 2: If the remaining SOC cannot afford the travel in the next day or $p(S O C)$ is true, the algorithm is launched to calculate $t_{r}, t_{c}$ and $t_{c, m i n}$ and $T_{s}$. $t_{r}$ represents the remaining time with the valley price of the TOU mechanism and it is calculated according to Formula A5. $t_{c}$ represents the time for charge a battery from its current SOC to full capacity. TL and TH are the start and end time points for the lower price period of the TOU. $T_{1}$ is the departure time in the next day and, if the EV is not used in the next day, $\mathrm{T}_{1}$ equals to $\mathrm{TH}$.

$S O C_{\text {day }}=\left(d_{t, 1}+\cdots+d_{t, n}\right) E / C$ 


$$
t_{r}= \begin{cases}24-\max (T, T L)+\min \left(T_{1}, T H\right) & T \geq T H \\ \min \left(T_{1}, T H\right)-T & T<T H\end{cases}
$$

A3. Basic information for the model

Table A2. Basic information of the EV

\begin{tabular}{|c|c|c|c|c|c|c|}
\hline EV & \multicolumn{2}{|c|}{$\begin{array}{l}\text { Battery Capacity } \\
\quad(k W h)\end{array}$} & \multicolumn{2}{|c|}{$\begin{array}{l}\text { Energy Consumption } \\
\quad(\mathrm{kWh} / \mathrm{km})\end{array}$} & \multicolumn{2}{|c|}{$\begin{array}{l}\text { Charging Efficiency } \\
\text { (\%) }\end{array}$} \\
\hline Nissan Leaf & \multicolumn{2}{|c|}{24} & \multicolumn{2}{|c|}{0.212} & \multicolumn{2}{|c|}{86.4} \\
\hline \multicolumn{7}{|c|}{ Table A3. Time-of-use pricing mechanism [35] } \\
\hline Time & $\begin{array}{l}0: 00- \\
7: 00\end{array}$ & $\begin{array}{l}7: 00- \\
10: 00\end{array}$ & $\begin{array}{l}10: 00- \\
13: 00\end{array}$ & $\begin{array}{l}13: 00- \\
18: 00\end{array}$ & $\begin{array}{l}18: 00- \\
23: 00\end{array}$ & $\begin{array}{l}23: 00- \\
24: 00\end{array}$ \\
\hline $\begin{array}{c}p_{\text {grid }} \\
\text { (RMB/kWh) }\end{array}$ & 0.475 & 0.900 & 1.346 & 0.900 & 1.346 & 0.475 \\
\hline
\end{tabular}




\section{Reference}

[1] Du J, Ouyang M, Chen J. Prospects for Chinese electric vehicle technologies in 2016-2020: Ambition and rationality. Energy. 2017; 120:584-96. Doi: https://doi.org/10.1016/j.energy.2016.11.114.

[2] Zhou B, Littler T, Meegahapola L, Zhang H. Power system steady-state analysis with large-scale electric vehicle integration. Energy. 2016; 115, Part 1:289-302. Doi: https://doi.org/10.1016/i.energy.2016.08.096.

[3] Božič D, Pantoš M. Impact of electric-drive vehicles on power system reliability. Energy. 2015; 83(Supplement C):511-20. Doi: https://doi.org/10.1016/j.energy.2015.02.055.

[4] Kavousi-Fard A, Abunasri A, Zare A, Hoseinzadeh R. Impact of plug-in hybrid electric vehicles charging demand on the optimal energy management of renewable micro-grids. Energy. 2014; 78:904-15. Doi: https://doi.org/10.1016/i.energy.2014.10.088.

[5] Anand MP, Golshannavaz S, Ongsakul W, Rajapakse A. Incorporating short-term topological variations in optimal energy management of MGs considering ancillary services by electric vehicles. Energy. 2016; 112:241-53. Doi: https://doi.org/10.1016/j.energy.2016.06.078.

[6] Muratori M. Impact of uncoordinated plug-in electric vehicle charging on residential power demand. Nature Energy. 2018; 3(3):193-201. (In English). Doi: 10.1038/s41560-017-0074-z.

[7] Wu XH, Hu XS, Teng YQ, Qian SD, Cheng R. Optimal integration of a hybrid solar-battery power source into smart home nanogrid with plug-in electric vehicle. Journal of Power Sources. 2017; 363:277-83. (In English). Doi: 10.1016/j.jpowsour.2017.07.086.

[8] Bian C, Li H, Wallin F, Avelin A, Lin L, Yu Z. Finding the optimal location for public charging stations a GIS-based MILP approach. Energy Procedia. 2019; 158:6582-8. Doi: https://doi.org/10.1016/j.egypro.2019.01.071.

[9] Perez HE, Hu X, Dey S, Moura SJ. Optimal Charging of Li-lon Batteries With Coupled Electro-ThermalAging Dynamics. IEEE Transactions on Vehicular Technology. 2017; 66(9):7761-70. Doi: 10.1109/TVT.2017.2676044.

[10] Zhang FQ, Hu XS, Langari R, Cao DP. Energy management strategies of connected HEVs and PHEVs: Recent progress and outlook. Progress in Energy and Combustion Science. 2019; 73:235-56. (In English). Doi: 10.1016/j.pecs.2019.04.002.

[11] Panwar LK, Reddy KS, Kumar R, Panigrahi BK, Vyas S. Strategic Energy Management (SEM) in a micro grid with modern grid interactive electric vehicle. Energy Conversion and Management. 2015; 106:4152. Doi: https://doi.org/10.1016/j.enconman.2015.09.019.

[12] Angrisani G, Canelli M, Roselli C, Sasso M. Integration between electric vehicle charging and microcogeneration system. Energy Conversion and Management. 2015; 98:115-26. Doi: https://doi.org/10.1016/j.enconman.2015.03.085.

[13] Drude L, Pereira LC, Ruther R. Photovoltaics (PV) and electric vehicle-to-grid (V2G) strategies for peak demand reduction in urban regions in Brazil in a smart grid environment. Renewable Energy. 2014; 68:443-51. (In English). Doi: 10.1016/j.renene.2014.01.049.

[14] Salpakari J, Rasku T, Lindgren J, Lund PD. Flexibility of electric vehicles and space heating in net zero energy houses: an optimal control model with thermal dynamics and battery degradation. Applied Energy. 2017; 190:800-12. (In English). Doi: 10.1016/j.apenergy.2017.01.005.

[15] Kavousi-Fard A, Khodaei A. Efficient integration of plug-in electric vehicles via reconfigurable microgrids. Energy. 2016; 111:653-63. Doi: https://doi.org/10.1016/i.energy.2016.06.018.

[16] Hoehne CG, Chester MV. Optimizing plug-in electric vehicle and vehicle-to-grid charge scheduling 
to minimize carbon emissions. Energy. 2016; 115, Part 1:646-57. Doi: https://doi.org/10.1016/j.energy.2016.09.057.

[17] Daina N, Sivakumar A, Polak JW. Modelling electric vehicles use: a survey on the methods. Renewable and Sustainable Energy Reviews. 2017; 68(Part 1):447-60. Doi: https://doi.org/10.1016/j.rser.2016.10.005.

[18] Fernandes C, Frias P, Latorre JM. Impact of vehicle-to-grid on power system operation costs: The Spanish case study. Applied Energy. 2012; 96:194-202. (In English). Doi: 10.1016/j.apenergy.2011.11.058.

[19] Zakariazadeh A, Jadid S, Siano P. Integrated operation of electric vehicles and renewable generation in a smart distribution system. Energy Conversion and Management. 2015; 89:99-110. Doi: https://doi.org/10.1016/j.enconman.2014.09.062.

[20] Jin X, Mu Y, Jia H, Wu J, Xu X, Yu X. Optimal day-ahead scheduling of integrated urban energy systems. Applied Energy. 2016; 180:1-13. Doi: https://doi.org/10.1016/i.apenergy.2016.07.071.

[21] Singh S, Jagota S, Singh M. Energy management and voltage stabilization in an islanded microgrid through an electric vehicle charging station. Sustainable Cities and Society. 2018; 41:679-94. Doi: https://doi.org/10.1016/j.scs.2018.05.055.

[22] Falahati S, Taher SA, Shahidehpour M. Grid frequency control with electric vehicles by using of an optimized fuzzy controller. Applied Energy. 2016; 178:918-28. Doi: https://doi.org/10.1016/j.apenergy.2016.06.077.

[23] Hashemi-Dezaki H, Hamzeh M, Askarian-Abyaneh H, Haeri-Khiavi H. Risk management of smart grids based on managed charging of PHEVs and vehicle-to-grid strategy using Monte Carlo simulation. Energy Conversion and Management. 2015; 100:262-76. Doi: https://doi.org/10.1016/j.enconman.2015.05.015.

[24] Schuller A, Flath CM, Gottwalt S. Quantifying load flexibility of electric vehicles for renewable energy integration. Applied Energy. 2015; 151:335-44. (In English). Doi: 10.1016/j.apenergy.2015.04.004.

[25] García-Villalobos J, Zamora I, Knezović K, Marinelli M. Multi-objective optimization control of plugin electric vehicles in low voltage distribution networks. Applied Energy. 2016; 180:155-68. Doi: https://doi.org/10.1016/j.apenergy.2016.07.110.

[26] Axsen, J., S. Goldberg, J. Bailey, G. Kamiya, B. Langman, J. Cairns, M. Wolinetz, and A. Miele (2015). Electrifying Vehicles: Insights from the Canadian Plug-in Electric Vehicle Study. Simon Fraser University, Vancouver, Canada.

[27] Lin H, Liu Y, Sun Q, Xiong R, Li H, Wennersten R. The impact of electric vehicle penetration and charging patterns on the management of energy hub-A multi-agent system simulation. Applied Energy. 2018; 230:189-206. Doi: https://doi.org/10.1016/j.apenergy.2018.08.083.

[28] Wang D, Gao J, Li P, Wang B, Zhang C, Saxena S. Modeling of plug-in electric vehicle travel patterns and charging load based on trip chain generation. Journal of Power Sources. 2017; 359:468-79. Doi: https://doi.org/10.1016/j.jpowsour.2017.05.036.

[29] Muratori M, Moran MJ, Serra E, Rizzoni G. Highly-resolved modeling of personal transportation energy consumption in the United States. Energy. 2013; 58:168-77. Doi: 10.1016/j.energy.2013.02.055. [30] U.S. Department of Transportation, Federal Highway Administration, 2017 National Household Travel Survey. URL: http://nhts.ornl.gov.

[31] Wen J, Tao S, Xiao X, Luo C, Liao K. Analysis on Charging Demand of EV Based on Stochastic Simulation of Trip Chain. Power System Technology. 2015; 39(6):1477-84. (In Chinese). 
[32] Brady J, O'Mahony M. Modelling charging profiles of electric vehicles based on real-world electric vehicle charging data. Sustainable Cities and Society. 2016; 26(Supplement C):203-16. Doi: https://doi.org/10.1016/j.scs.2016.06.014.

[33] Flores RJ, Shaffer BP, Brouwer J. Electricity costs for an electric vehicle fueling station with Level 3 charging. Applied Energy. 2016; 169:813-30. Doi: https://doi.org/10.1016/j.apenergy.2016.02.071.

[34] Pan Z, Wu J, Sun H, Abeysekera M. Quantification of Operational Flexibility from a Heating Network. Energy Procedia. 2018; 145:516-21. Doi: https://doi.org/10.1016/i.egypro.2018.04.075.

[35] Lin H, Wang Q, Wang Y, Liu Y, Huang N, Wennersten R, Sun Q. A multi-agent based optimization architecture for energy hub operation. Energy Procedia. 2017; 142:2158-64. Doi: https://doi.org/10.1016/j.egypro.2017.12.621.

[36] Lin H, Bian C, Li H, Sun Q, Wennersten R. Optimal siting and sizing of public charging stations in urban area. In: International Conference on Electric and Intelligent Vehicles; November 21-25, 2018; Melbourne, Australia. 2018.

[37] Mavromatidis G, Orehounig K, Carmeliet J. Uncertainty and global sensitivity analysis for the optimal design of distributed energy systems. Applied Energy. 2018; 214:219-38. Doi: https://doi.org/10.1016/j.apenergy.2018.01.062.

[38] Battery electric vehicle driving and charging behavior observed early in the EV project. 2012. SAE International. 